\title{
Molecular ions in the protostellar shock L1157-B1 ${ }^{\star}$
}

\author{
L. Podio ${ }^{1,2}$, B. Lefloch ${ }^{1}$, C. Ceccarelli ${ }^{1}$, C. Codella ${ }^{2}$, and R. Bachiller ${ }^{3}$ \\ 1 Institut de Planétologie et d'Astrophysique de Grenoble, 414 rue de la Piscine, 38400 St-Martin d'Hères, France \\ e-mail: lpodio@arcetri.astro.it \\ 2 INAF - Osservatorio Astrofisico di Arcetri, Largo E. Fermi 5, 50125 Firenze, Italy \\ 3 IGN Observatorio Astronómico Nacional, Apartado 1143, 28800 Alcalá de Henares, Spain
}

Received 28 October 2013 / Accepted 8 February 2014

\begin{abstract}
Aims. We perform a complete census of molecular ions with an abundance greater than $\sim 10^{-10}$ in the protostellar shock L1157-B1. This allows us to study the ionisation structure and chemistry of the shock.

Methods. An unbiased high-sensitivity survey of L1157-B1 performed with the IRAM-30 m and Herschel/HIFI as part of the CHESS and ASAI large programmes allows searching for molecular ions emission. Then, by means of a radiative transfer code in the large velocity gradient approximation, the gas physical conditions and fractional abundances of molecular ions are derived. The latter are compared with estimates of steady-state abundances in the cloud and their evolution in the shock calculated with the chemical model Astrochem.

Results. We detect emission from $\mathrm{HCO}^{+}, \mathrm{H}^{13} \mathrm{CO}^{+}, \mathrm{N}_{2} \mathrm{H}^{+}, \mathrm{HCS}^{+}$, and for the first time in a shock, from $\mathrm{HOCO}^{+}$and $\mathrm{SO}^{+}$. The bulk of the emission peaks at blue-shifted velocity, $\sim 0.5-3 \mathrm{~km} \mathrm{~s}^{-1}$ with respect to systemic, has a width of $\sim 3-7 \mathrm{~km} \mathrm{~s}^{-1}$ and is associated with the outflow cavities $\left(T_{\text {kin }} \sim 20-70 \mathrm{~K}, n_{\mathrm{H}_{2}} \sim 10^{5} \mathrm{~cm}^{-3}\right)$. A high-velocity component up to $-40 \mathrm{~km} \mathrm{~s}^{-1}$, associated with the primary jet, is detected in the $\mathrm{HCO}^{+} 1-0$ line. Observed $\mathrm{HCO}^{+}$and $\mathrm{N}_{2} \mathrm{H}^{+}$abundances $\left(X_{\mathrm{HCO}^{+}} \sim 0.7-3 \times 10^{-8}, X_{\mathrm{N}_{2} \mathrm{H}^{+}} \sim 0.4-8 \times 10^{-9}\right)$ agree with steady-state abundances in the cloud and with their evolution in the compressed and heated gas in the shock for cosmic rays ionisation rate $\zeta=3 \times 10^{-16} \mathrm{~s}^{-1}$. $\mathrm{HOCO}^{+}, \mathrm{SO}^{+}$, and $\mathrm{HCS}^{+}$observed abundances $\left(X_{\mathrm{HOCO}^{+}} \sim 10^{-9}, X_{\mathrm{SO}^{+}} \sim 8 \times 10^{-10}, X_{\mathrm{HCS}^{+}} \sim 3-7 \times 10^{-10}\right)$, instead, are 1-2 orders of magnitude larger than predicted in the cloud; on the other hand, they are strongly enhanced on timescales shorter than the shock age ( 2000 years) if $\mathrm{CO}_{2}, \mathrm{~S}$ or $\mathrm{H}_{2} \mathrm{~S}$, and OCS are sputtered off the dust grains in the shock.

Conclusions. The performed analysis indicates that $\mathrm{HCO}^{+}$and $\mathrm{N}_{2} \mathrm{H}^{+}$are a fossil record of pre-shock gas in the outflow cavity, whilst $\mathrm{HOCO}^{+}, \mathrm{SO}^{+}$, and $\mathrm{HCS}^{+}$are effective shock tracers that can be used to infer the amount of $\mathrm{CO}_{2}$ and sulphur-bearing species released from dust mantles in the shock. The observed $\mathrm{HCS}^{+}$(and CS) abundance indicates that OCS should be one of the main sulphur carrier on grain mantles. However, the OCS abundance required to fit the observations is 1-2 orders of magnitude larger than observed. Laboratory experiments are required to measure the reactions rates involving these species and to fully understand the chemistry of sulphur-bearing species.
\end{abstract}

Key words. stars: formation - ISM: jets and outflows - ISM: molecules - ISM: abundances - astrochemistry

\section{Introduction}

The gravitational infall from which a new star is formed is accompanied by the ejection of highly supersonic jets. The shocks produced when the ejected material impacts on the high-density surrounding medium rapidly heat and compress the gas, triggering several microscopic processes, such as molecular dissociation, gas ionisation, endothermic chemical reactions, ice sublimation, and dust grain disruption. It is well known that these processes produce a strong enhancement of the abundance of some molecules, which are evaporated or sputtered from dust grains, such as $\mathrm{H}_{2} \mathrm{CO}, \mathrm{CH}_{3} \mathrm{OH}, \mathrm{NH}_{3}, \mathrm{SiO}$ (see, e.g., Bachiller \& Perez Gutierrez 1997).

Molecular ions are commonly observed in dense prestellar/protostellar regions (e.g., Caselli et al. 1998, 2002; Hogerheijde et al. 1998), and their chemistry has been studied

* The molecular ions line spectra shown in Fig. 2 are only available at the CDS via anonymous ftp to

cdsarc.u-strasbg.fr (130.79.128.5) or via

http://cdsarc.u-strasbg.fr/viz-bin/qcat?]/A+A/565/A64 by means of gas-phase chemical models (e.g., Herbst \& Leung 1986; Turner et al. 1999). In contrast, there are very few observations of molecular ions in protostellar shocks. $\mathrm{HCO}^{+}$emission was detected in a number of outflows, and in some cases it shows a butterfly morphology that suggests excitation in the outflow cavities (e.g., Hogerheijde et al. 1998; Girart et al. 1999, 2002; Lee et al. 2007; Tafalla et al. 2010; Tappe et al. 2012). Besides $\mathrm{HCO}^{+}$, emission in $\mathrm{HCS}^{+}$and, very recently, in $\mathrm{N}_{2} \mathrm{H}^{+}$lines has been detected in the $\mathrm{B} 1$ shock along the chemically rich $\mathrm{L} 1157$ outflow (Bachiller \& Perez Gutierrez 1997; Yamaguchi et al. 2012; Codella et al. 2013).

The lack of observations means that the chemical evolution of molecular ions in shocks has been poorly investigated. On the one hand, molecular ions are expected to be rapidly destroyed by electronic recombination in shocks; on the other, they may be enhanced by the sputtering of dust grain mantles, which inject molecules into the gas phase where they undergo reactions leading to ions (e.g., Neufeld \& Dalgarno 1989; Viti et al. 2002; Rawlings et al. 2004). Among the molecular ions previously observed in shocks, not all of them are due to changes caused by 
the shock itself. Codella et al. (2013) show that the $\mathrm{N}_{2} \mathrm{H}^{+}$abundance is expected to decrease in shocks, so the emission detected in the L1157-B1 shock is a probe of pre-shock chemistry. Molecular ions such as $\mathrm{HOCO}^{+}$and $\mathrm{SO}^{+}$are instead believed to be effective tracers of shocked gas, since their abundance is predicted to be very low in the quiescent gas $\left(\leq 10^{-10}\right)$ but could be strongly enhanced in shocks following the release of $\mathrm{CO}_{2}$ and S-bearing molecules from dust grains mantles (e.g., Minh et al. 1991; Turner 1992, 1994; Deguchi et al. 2006). However, there are no observations of $\mathrm{HOCO}^{+}$and $\mathrm{SO}^{+}$undoubtedly associated with shocks.

In this paper, a complete census of molecular ions in a shock is given for the first time. This is obtained by means of a highly-sensitive unbiased spectral survey of the prototypical protostellar bow shock L1157-B1 in the millimetre and submillimetre ranges, executed with the IRAM-30 m telescope as part of the ASAI large programme ${ }^{1}$ (Astrochemical Surveys At Iram, Lefloch et al., in prep.) and with Herschel/HIFI as part of the guaranteed time key project CHESS $^{2}$ (Chemical HErschel Surveys of Star forming regions, Ceccarelli \& CHESS Consortium 2010). The observations are presented in Sect. 2, whilst Sect. 3 summarises the main properties of the L1157 outflow and the B1 bow shock. In Sect. 4 we analyse the line profiles of the detected molecular ions and perform a large velocity gradient (LVG) analysis to infer the physical conditions of the emitting gas and the abundance of molecular ions. Finally, in Sect. 5 we compare the observed abundances with steadystate abundances in the cloud and their evolution in the shock computed using the chemical code Astrochem. This allows us to distinguish between molecular ions that are tracing pre-shock chemistry and those that are useful probes of the shock properties and chemistry. Our conclusions are summarised in Sect. 6.

\section{Observations and data reduction}

\subsection{IRAM-30 m observations}

The millimetre observations of L1157-B1 were acquired with the IRAM-30 m telescope at Pico Veleta (Spain), as part of the ASAI large programme (Lefloch, in prep.). The observed position in B1 is at $\alpha_{\mathrm{J} 2000}=20^{\mathrm{h}} 39^{\mathrm{m}} 10^{\mathrm{s}} .2, \delta_{\mathrm{J} 2000}=+68^{\circ} 01^{\prime} 10^{\prime} .5$. The survey, obtained during several runs in 2011 and 2012, covers the spectral bands at $3 \mathrm{~mm}(80-116 \mathrm{GHz}), 2 \mathrm{~mm}(128-173 \mathrm{GHz})$, $1.3 \mathrm{~mm}(200-320 \mathrm{GHz})$, and $0.8 \mathrm{~mm}(328-350 \mathrm{GHz})$. The observations were carried out in wobbler switching mode, with a throw of $3^{\prime}$, and with the broad-band EMIR receivers and the FTS spectrometers in its $200 \mathrm{kHz}$ resolution mode, corresponding to velocity resolutions of $0.17-0.75 \mathrm{~km} \mathrm{~s}^{-1}$. The half power beam width (HPBW) varies between $7^{\prime \prime}$ at $350 \mathrm{GHz}$ and $31^{\prime \prime}$ at $80 \mathrm{GHz}$. The data were processed using GILDAS/CLASS90 3 software. The spectra were smoothed by a factor of two, baseline-subtracted, and further resampled at lower spectral resolution if needed to increase the signal-to-noise. The rms achieved is typically $1.5 \mathrm{mK}$ per interval of $0.4 \mathrm{MHz}$ (i.e., $\sim 1.4 \mathrm{~km} \mathrm{~s}^{-1}$ ) across the $3 \mathrm{~mm}$ band and $\sim 5 \mathrm{mK}$ per interval of $\sim 1 \mathrm{~km} \mathrm{~s}^{-1}$ in the $2 \mathrm{~mm}$ and $1.3 \mathrm{~mm}$ bands. Line intensities are expressed in units of main-beam temperature, $T_{\mathrm{mb}}$. We adopted the main-beam efficiencies, $\eta_{\mathrm{mb}}$, monitored by IRAM

\footnotetext{
1 http://www.oan.es/asai

2 http://chess.obs.ujf-grenoble.fr

3 http://www.iram. fr/IRAMFR/GILDAS
}

(http: //www . iram.fr). These decrease from 0.82 at $80 \mathrm{GHz}$ to 0.33 in the sub-mm range.

\subsection{Herschel/HIFl observations}

The IRAM-30 m survey is complemented by observations in the sub-millimetre range taken with the Heterodyne Instrument for the Far Infrared (HIFI, de Graauw et al. 2010) on board the Herschel Space Observatory ${ }^{4}$ (Pilbratt et al. 2010) as part of the guaranteed time key project CHESS (Ceccarelli \& CHESS Consortium 2010). The observations were carried out during 2010 towards the nominal position of B1 and in dual beam switch spectral scanning mode to cover most of the submillimetre bands 1 and 2 (488-628 GHz, 642-794 GHz). Both polarisations ( $\mathrm{H}$ and $\mathrm{V}$ ) were observed simultaneously. The receiver was tuned in double sideband (DSB) and the Wide Band Spectrometer (WBS) was used, providing a spectral resolution of $1.1 \mathrm{MHz}$, corresponding to $0.5-0.7 \mathrm{~km} \mathrm{~s}^{-1}$, which was subsequently degraded to increase the sensitivity. The data were processed with the ESA-supported package Herschel Interactive Processing Environment ${ }^{5}$ (HIPE, Ott 2010) version 6 package. Then level 2 fits files were transformed into GILDAS format for baseline subtraction and subsequent sideband deconvolution. The relative calibration between both receivers ( $\mathrm{H}$ and $\mathrm{V}$ ) was found to be very good, and the signals were co-added to improve the signal-to-noise. Line intensities were converted from antenna temperature, $T_{\mathrm{a}}$, to main-beam brightness temperature, $T_{\mathrm{mb}}$, using the main-beam efficiency, $\eta_{\mathrm{mb}}$, as determined by Roelfsema et al. (2012).

\section{Previous work on L1157}

The L1157 outflow, located at a distance of $\sim 250$ pc (Looney et al. 2007) and driven by the low mass Class 0 protostar L1157-mm $\left(\sim 4 L_{\odot}\right)$, is the most chemically rich outflow known so far (e.g., Bachiller \& Perez Gutierrez 1997; Bachiller et al. 2001). Observations in $\mathrm{CO}, \mathrm{H}_{2}$, and $\mathrm{H}_{2} \mathrm{O}$ lines reveal a highly collimated and precessing bipolar outflow (Davis \& Eisloeffel 1995; Gueth et al. 1996), associated with several blue-shifted (B0, B1, B2) and red-shifted (R0, R1, R, R2) bow shocks (Bachiller et al. 2001; Nisini et al. 2010) (see top panel of Fig. 1). Spectral surveys also revealed emission in molecules that are released from dust mantles, such as $\mathrm{SO}, \mathrm{H}_{2} \mathrm{~S}, \mathrm{OCS}, \mathrm{H}_{2} \mathrm{CO}$, $\mathrm{CH}_{3} \mathrm{OH}, \mathrm{H}_{2} \mathrm{O}$, and $\mathrm{NH}_{3}$ (Tafalla \& Bachiller 1995; Bachiller \& Perez Gutierrez 1997; Benedettini et al. 2007; Codella et al. 2010; Vasta et al. 2012), or sputtered from their core, such as $\mathrm{SiO}$ (Gueth et al. 1998; Nisini et al. 2007).

The brightest bow shock B1 is an ideal laboratory for investigating the effect of shocks on gas chemistry because it is located at $\sim 69^{\prime \prime}$ from the protostar $(\sim 0.1 \mathrm{pc}$, see bottom panel of Fig. 1$)$, so UV irradiation can be neglected. The first results obtained from our IRAM-30 m/Herschel survey further highlighted the chemical richness of B1 (Codella et al. 2010, 2012a,b; Lefloch et al. 2010; Benedettini et al. 2012; Busquet et al. 2014). High angular resolution interferometric observations reveal that B1 has a clumpy structure with multiple components (Benedettini et al. 2007; Codella et al. 2009; Benedettini et al. 2013),

\footnotetext{
4 Herschel is an ESA space observatory with science instruments provided by European-led Principal Investigator consortia and with important participation from NASA.

5 HIPE is a joint development by the Herschel Science Ground Segment Consortium, consisting of ESA, the NASA Herschel Science Center, and the HIFI, PACS and SPIRE consortia.
} 


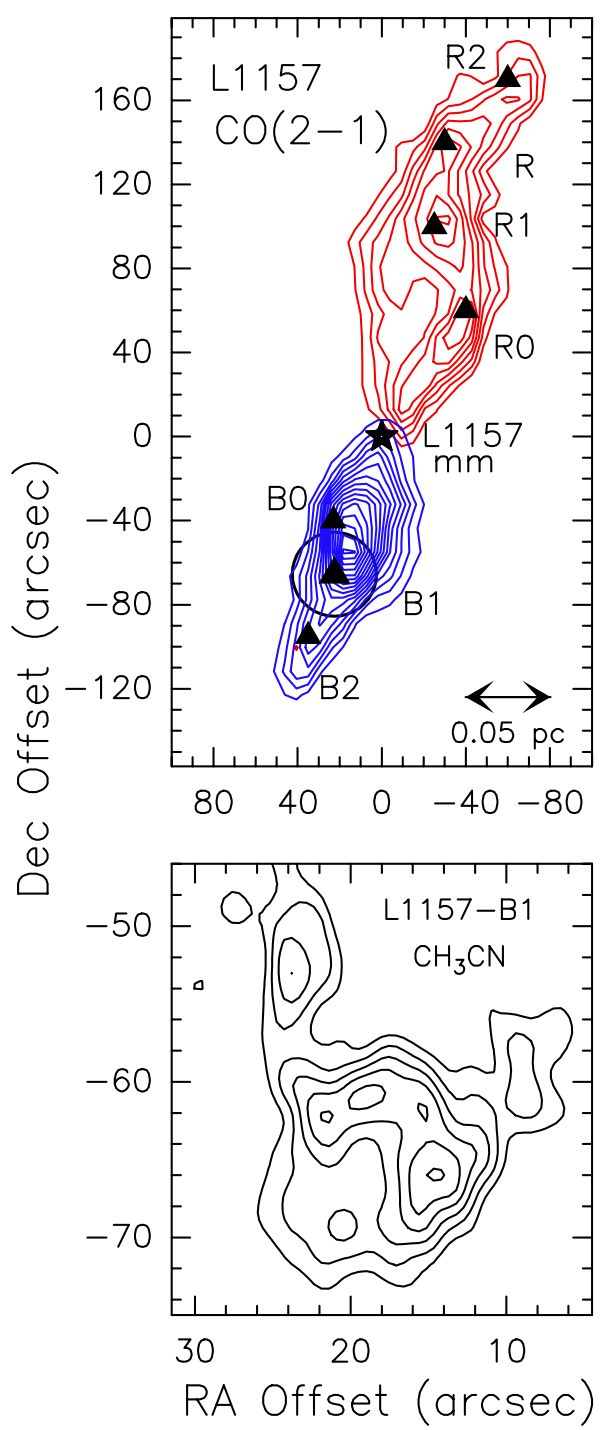

Fig. 1. Top panel: integrated CO $J=2-1$ emission of the L1157 bipolar outflow (Bachiller et al. 2001). Offsets are with respect to the driving source L1157-mm (black star), at coordinates: $\alpha_{\mathrm{J} 2000}=20^{\mathrm{h}} 39^{\mathrm{m}} 06^{\mathrm{s}} .2$, $\delta_{\mathrm{J} 2000}=+68^{\circ} 02^{\prime} 16^{\prime \prime}$. $^{\prime}$. The black triangles and labels indicate the main blue- and red-shifted knots as defined by Bachiller et al. (2001). Circle is for the largest HPBW of the present dataset $\left(40^{\prime \prime}\right)$, centred on the L1157-B1 bow shock. Bottom panel: the L1157-B1 bow shock as traced by the $\mathrm{CH}_{3} \mathrm{CN} J=8-7 \mathrm{~K}=0,1,2$ emission at $3 \mathrm{~mm}$, observed with the IRAM PdB interferometer (Codella et al. 2009).

which cannot be resolved by our single-dish IRAM-30 m and Herschel/HIFI observations. However, Lefloch et al. (2012) showed that the analysis of the line profiles provides a simple tool for identifying the different spatial and velocity components. In particular, the CO line profiles from $J=1-0$ to $J=16-15$ are well fitted by a linear combination of three velocity components $\left(g_{1}, g_{2}\right.$, and $\left.g_{3}\right)$, whose intensity-velocity relation follows an exponential law $I(V)=I(0) \exp \left(-\left|V / V_{0}\right|\right)$, with $V_{0}=12.5$, 4.4 , and $2.5 \mathrm{~km} \mathrm{~s}^{-1}$ for $g_{1}, g_{2}$, and $g_{3}$, respectively. These velocity components trace different regions in the B1 bow shock, characterized by different sizes and physical conditions:

(i) $g_{1}$ : the jet impact region against the cavity $\left(\sim 7-10^{\prime \prime}\right)$ in the L1157-B1 bow shock, with gas at $T_{\text {kin }} \sim 210 \mathrm{~K}$, reaching velocities up to $-40 \mathrm{~km} \mathrm{~s}^{-1}$;

(ii) $g_{2}$ : the outflow cavity associated with B1, made of gas at $T_{\text {kin }} \sim 60-80 \mathrm{~K}$, reaching velocities up to $-20 \mathrm{~km} \mathrm{~s}^{-1}$; (iii) $g_{3}$ : the cavity associated with the older outflow shock L1157-B2, made of colder gas $\left(T_{\text {kin }} \sim 20 \mathrm{~K}\right)$ at slower velocities $\left(\leq-10 \mathrm{~km} \mathrm{~s}^{-1}\right)$.

Besides the three components shown by the analysis of the CO line profiles, a fourth hot and tenuous component $\left(T_{\text {kin }} \sim\right.$ $1000 \mathrm{~K}, n_{\mathrm{H}_{2}} \sim 10^{4} \mathrm{~cm}^{-3}$ ) is detected in $\mathrm{H}_{2} \mathrm{O}$ lines (Busquet et al. 2014).

Interestingly, L1157-B1 also shows emission in molecular ions, such as $\mathrm{HCO}^{+}, \mathrm{HCS}^{+}$, and $\mathrm{N}_{2} \mathrm{H}^{+}$(Bachiller \& Perez Gutierrez 1997; Codella et al. 2010, 2013; Yamaguchi et al. 2012), making it a unique target for investigating the chemistry of molecular ions in protostellar shocks.

\section{Results}

\subsection{Line identification}

The ions identified down to the $3 \sigma$ level $(\sim 5 \mathrm{mK}$ in the $3 \mathrm{~mm}$ band) in $\mathrm{L} 1157-\mathrm{B} 1$ are $\mathrm{HCO}^{+}, \mathrm{H}^{13} \mathrm{CO}^{+}, \mathrm{N}_{2} \mathrm{H}^{+}, \mathrm{HOCO}^{+}, \mathrm{SO}^{+}$, and $\mathrm{HCS}^{+}$. Thanks to the high sensitivity of our survey, several transitions of each molecular species are detected, allowing high accuracy in the derivation of the physical conditions. $\mathrm{HOCO}^{+}$ and $\mathrm{SO}^{+}$are observed for the first time in L1157-B1 and, more in general, in a protostellar shock.

The properties of the detected lines (transition, frequency $\left(v_{0}\right)$ in $\mathrm{MHz}$, upper level energy $\left(E_{\mathrm{up}}\right)$ in $\left.\mathrm{K}\right)$ and their observational parameters (telescope half power beam width (HPBW) in arcseconds, rms noise in $\mathrm{mK}$, peak velocity $\left(V_{\text {peak }}\right)$ in $\mathrm{km} \mathrm{s}^{-1}$, peak temperature $\left(T_{\text {peak }}\right)$ in main-beam temperature units, full width at half maximum (FWHM) in $\mathrm{km} \mathrm{s}^{-1}$, and integrated intensity $\left(\int T_{\mathrm{mb}} \mathrm{d} V\right)$ in $\left.\mathrm{K} \mathrm{km} \mathrm{s}^{-1}\right)$ are summarised in Table 1. All the transitions reported in the table are observed with the IRAM- $30 \mathrm{~m} / \mathrm{FTS}$, except those indicated by the star which are observed with Herschel/HIFI. A Gaussian fit is applied to estimate the line properties for most of the transitions. When the line profile is non-Gaussian the line intensity is obtained by integrating the area below the profile. For each species we also report a $3 \sigma$ upper limit for the first non-detected transition covered by our observations. The line spectra are shown in Fig. 2. All the detected lines peak at blue-shifted velocity, $\sim 0.5-3 \mathrm{~km} \mathrm{~s}^{-1}$ with respect to systemic, and have a line width of $\sim 3-7 \mathrm{~km} \mathrm{~s}^{-1}$, which is consistent with emission originating in the outflow cavities B1 and B2. A detailed analysis of the observed line profiles for each molecular ion is presented in the following sections.

\subsection{Physical conditions and abundances}

The gas physical conditions and the column density and abundance of each molecular species were derived from a multitransition analysis, by means of a radiative transfer code in the LVG approximation whenever more than one line is detected and the collisional coefficients are available $\left(\mathrm{HCO}^{+}, \mathrm{HOCO}^{+}\right.$, and $\mathrm{HCS}^{+}$), and in the hypothesis of local thermodynamic equilibrium (LTE) otherwise $\left(\mathrm{N}_{2} \mathrm{H}^{+}\right.$, and $\left.\mathrm{SO}^{+}\right)$. The Einstein coefficients and upper level energies were retrieved from the Jet Propulsion Laboratory (JPL) database ${ }^{6}$ (Pickett et al. 1998) and the Cologne Database of Molecular Spectroscopy (CDMS) ${ }^{7}$ (Müller et al. 2001), whilst the collisional coefficients were taken from the BASECOL database ${ }^{8}$ (Dubernet et al. 2013). In particular, the references for the collisional coefficients are Flower (1999) for $\mathrm{HCO}^{+}$, Hammami et al. (2007) for $\mathrm{HOCO}^{+}$,

\footnotetext{
6 http://spec.jpl.nasa.gov

7 http://www .astro.uni-koeln.de/cdms

8 http://basecol.obspm.fr/
} 
Table 1. Properties of the detected lines from molecular ions.

\begin{tabular}{|c|c|c|c|c|c|c|c|c|c|}
\hline Species & Transition & $\begin{array}{c}v_{0}{ }^{a} \\
(\mathrm{MHz})\end{array}$ & $\begin{array}{l}E_{\text {up }} \\
(\mathrm{K})\end{array}$ & $\begin{array}{c}\text { HPBW } \\
\left({ }^{\prime \prime}\right)\end{array}$ & $\begin{array}{l}\mathrm{rms} \\
(\mathrm{mK})\end{array}$ & $\begin{array}{c}V_{\text {peak }} \\
\left(\mathrm{km} \mathrm{s}^{-1}\right)\end{array}$ & $\begin{array}{c}F W H M \\
\left(\mathrm{~km} \mathrm{~s}^{-1}\right)\end{array}$ & $\begin{array}{l}T_{\text {peak }} \\
(\mathrm{mK})\end{array}$ & $\begin{array}{c}\int_{\left(\mathrm{K} \mathrm{km} \mathrm{s}{ }^{-1}\right)} T_{\mathrm{mb}} \mathrm{d} V \\
\end{array}$ \\
\hline \multirow{5}{*}{$\mathrm{HCO}^{+}$} & $J=1-0^{\mathrm{ng}}$ & 89188.52 & 4 & 28 & 1 & $1.1 \pm 1.5$ & $4.4 \pm 1.5$ & $1095 \pm 1$ & $7.40 \pm 0.01$ \\
\hline & $J=3-2^{\text {ng }}$ & 267557.53 & 26 & 9 & 14 & $1.2 \pm 1.5$ & $6.0 \pm 1.5$ & $901 \pm 14$ & $5.49 \pm 0.09$ \\
\hline & $J=6-5^{*}$ & 535061.38 & 90 & 40 & 7 & $1.6 \pm 0.2$ & $4.6 \pm 0.4$ & $76 \pm 7$ & $0.38 \pm 0.03$ \\
\hline & $J=7-6^{*}$ & 624208.19 & 120 & 34 & 9 & $0.5 \pm 0.4$ & $3.5 \pm 0.8$ & $37 \pm 9$ & $0.14 \pm 0.03$ \\
\hline & $J=8-7^{*}$ & 713342.12 & 154 & 30 & 35 & - & - & - & $\leq 0.29$ \\
\hline \multirow{3}{*}{$\mathrm{H}^{13} \mathrm{CO}^{+}$} & $J=1-0$ & 86754.29 & 4 & 28 & 2 & $1.2 \pm 0.1$ & $3.7 \pm 0.2$ & $39 \pm 2$ & $0.15 \pm 0.00$ \\
\hline & $J=3-2$ & 260255.34 & 25 & 9 & 7 & $1.6 \pm 0.3$ & $1.8 \pm 0.7$ & $23 \pm 7$ & $0.04 \pm 0.01$ \\
\hline & $J=4-3$ & 346998.34 & 42 & 7 & 29 & - & - & - & $\leq 0.16$ \\
\hline \multirow[t]{2}{*}{$\mathrm{N}_{2} \mathrm{H}^{+b}$} & $J=1-0$ & 93173.76 & 5 & 26 & 2 & $1.3 \pm 0.1$ & $4.3 \pm 0.2$ & $29 \pm 2$ & $0.33 \pm 0.01$ \\
\hline & $J=3-2$ & 279511.83 & 27 & 9 & 5 & - & - & - & $\leq 0.03$ \\
\hline \multirow[t]{3}{*}{$\mathrm{HOCO}^{+c}$} & $J_{K_{-1} K_{1}}=4_{04}-3_{03} \mathrm{ng}$ & 85531.51 & 10 & 29 & 1 & $0.9 \pm 1.4$ & $6.8 \pm 1.4$ & $8 \pm 1$ & $0.06 \pm 0.01$ \\
\hline & $\mathrm{J}_{K_{-1} K_{1}}=5_{05}-4_{04}{ }^{\mathrm{ng}}$ & 106913.57 & 15 & 23 & 2 & $1.0 \pm 1.1$ & $3.3 \pm 1.1$ & $12 \pm 2$ & $0.04 \pm 0.01$ \\
\hline & $\mathrm{J}_{K_{-1} K_{1}}=6_{06}-5_{05}$ & 128295.06 & 22 & 19 & 11 & - & - & - & $\leq 0.07$ \\
\hline \multirow[t]{8}{*}{$\mathrm{SO}^{+d}$} & ${ }^{2} \Pi_{1 / 2}, J=5 / 2-3 / 2,1=\mathrm{e}$ & 115804.41 & 9 & 21 & 13 & - & - & - & $\leq 0.09$ \\
\hline & ${ }^{2} \Pi_{1 / 2}, J=5 / 2-3 / 2,1=\mathrm{f}$ & 116179.95 & 9 & 21 & 15 & - & - & - & $\leq 0.12$ \\
\hline & ${ }^{2} \Pi_{1 / 2}, J=7 / 2-5 / 2,1=\mathrm{e}^{\mathrm{ng}}$ & 162198.59 & 17 & 15 & 5 & $2.0 \pm 1.0$ & $2.9 \pm 1.0$ & $13 \pm 5$ & $0.09 \pm 0.02$ \\
\hline & ${ }^{2} \Pi_{1 / 2}, J=7 / 2-5 / 2,1=\mathrm{f}$ & 162574.06 & 17 & 15 & 7 & $0.6 \pm 0.8$ & $6.3 \pm 1.5$ & $17 \pm 7$ & $0.12 \pm 0.03$ \\
\hline & ${ }^{2} \Pi_{1 / 2}, J=9 / 2-7 / 2,1=\mathrm{e}$ & 208590.03 & 27 & 12 & 4 & $-0.2 \pm 0.6$ & $5.7 \pm 1.7$ & $13 \pm 4$ & $0.08 \pm 0.02$ \\
\hline & ${ }^{2} \Pi_{1 / 2}, J=9 / 2-7 / 2,1=\mathrm{f}$ & 208965.42 & 27 & 12 & 4 & $1.3 \pm 0.7$ & $5.6 \pm 2.0$ & $13 \pm 4$ & $0.08 \pm 0.02$ \\
\hline & ${ }^{2} \Pi_{1 / 2}, J=11 / 2-9 / 2,1=\mathrm{e}$ & 254977.94 & 39 & 10 & 5 & - & - & - & $\leq 0.03$ \\
\hline & ${ }^{2} \Pi_{1 / 2}, J=11 / 2-9 / 2,1=\mathrm{f}$ & 255353.23 & 39 & 10 & 5 & - & - & - & $\leq 0.03$ \\
\hline \multirow[t]{5}{*}{$\mathrm{HCS}^{+}$} & $J=2-1$ & 85347.90 & 6 & 29 & 1 & $-0.3 \pm 0.8$ & $6.2 \pm 1.9$ & $23 \pm 1$ & $0 . \overline{15} \pm 0.04$ \\
\hline & $J=4-3$ & 170691.73 & 21 & 14 & 7 & $0.9 \pm 0.3$ & $5.6 \pm 0.7$ & $36 \pm 7$ & $0.21 \pm 0.02$ \\
\hline & $J=5-4$ & 213360.53 & 31 & 12 & 4 & $0.0 \pm 0.2$ & $6.2 \pm 0.5$ & $38 \pm 4$ & $0.25 \pm 0.02$ \\
\hline & $J=6-5$ & 256027.80 & 43 & 10 & 6 & $1.1 \pm 0.3$ & $6.9 \pm 0.7$ & $30 \pm 6$ & $0.22 \pm 0.02$ \\
\hline & $J=7-6$ & 298690.88 & 57 & 8 & 16 & - & - & - & $\leq 0.09$ \\
\hline
\end{tabular}

Notes. ${ }^{(*)}$ Observed with Herschel/HIFI. ${ }^{(n g}$ ) Non-Gaussian profile. The line intensity is obtained by integrating the area below the profile. (a) Frequencies are from the JPL molecular database (Pickett et al. 1998), and from the CDMS database (Müller et al. 2001) for $\mathrm{N}_{2} \mathrm{H}^{+} .{ }^{(b)} \mathrm{The}$ line properties quoted for the $\mathrm{N}_{2} \mathrm{H}^{+} 1-0$ and 3-2 transitions refer to the brightest of their 15 and 45 hyperfine components, i.e. the $F 1, F=2$,

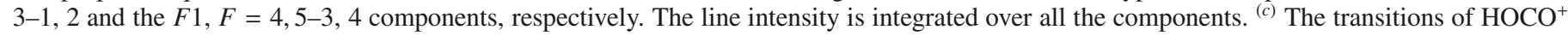
are characterized by the quantum numbers $J$, which is the total angular momentum, and $K_{-1}, K_{1}$, which are the angular momenta around the molecule's symmetry axis. ${ }^{(d)} \mathrm{SO}^{+}$is a reactive radical with ${ }^{2} \Pi_{1 / 2}$ ground state, whose spin-doubled rotational transitions, characterized by the quantum number $J$, are further split by $\Lambda$-type doubling.

and Monteiro (1984) for $\mathrm{HCS}^{+}$. In the case where an LTE analysis was applied, the main source of uncertainty on the derived abundance is the assumed excitation temperature and size of the emitting region. This leads to an uncertainty of up to an order of magnitude for $\mathrm{N}_{2} \mathrm{H}^{+}$, and about a factor three for $\mathrm{SO}^{+}$, for which the excitation temperature is derived by fitting the detected transitions. When, instead, an LVG analysis is applied the uncertainty on the abundance is obtained by considering the difference between the best-fit and the second best-fit solutions and is around a factor three. The gas kinetic temperature and $\mathrm{H}_{2}$ density, $T_{\text {kin }}$ and $n_{\mathrm{H}_{2}}$, the column density, $N_{\text {species }}$, and fractional abundances with respect to $\mathrm{H}_{2}, X_{\text {species }}=N_{\text {species }} / N_{\mathrm{H}_{2}}$, derived for the analysed molecular ions are summarised in Table 2.

\subsection{1. $\mathrm{HCO}^{+}$and isotopologues}

Figures $2 \mathrm{a}$ and $\mathrm{d}$ show all the detected transition from $\mathrm{HCO}^{+}$ and its isotopologue $\mathrm{H}^{13} \mathrm{CO}^{+}$. $\mathrm{HCO}^{+}$has been widely detected in prestellar cores (e.g., Caselli et al. 1998), in embedded low-mass protostars, and along their outflows (e.g., Hogerheijde et al. 1998). $\mathrm{HCO}^{+}$emission is detected up to the $J=7-6$ transition. We also detect emission from $\mathrm{H}^{13} \mathrm{CO}^{+}$in the $1-0$ and, tentatively, in the 3-2 line (the latter with a signal-to-noise of 4). The $\mathrm{HC}^{18} \mathrm{O}^{+} 1-0$ and 3-2 lines, instead, are not detected. The $\mathrm{HCO}^{+} 1-0$ line shows bright and very broad wings extending up to $-40 \mathrm{~km} \mathrm{~s}^{-1}$. So far, only $\mathrm{CO}, \mathrm{H}_{2} \mathrm{O}$, and $\mathrm{SiO}$ have been observed at these extreme velocities in L1157-B1 (Lefloch et al. 2010, 2012). High-velocity emission in $\mathrm{HCO}^{+}$lines has also been detected in the outflows driven by the Class 0 protostars L1448-mm and IRAS 04166+2706 (Tafalla et al. 2010).

The $\mathrm{HCO}^{+} 1-0$ line optical depth is derived from the $\mathrm{HCO}^{+}$ to the $\mathrm{H}^{13} \mathrm{CO}^{+} 1-0$ line ratio assuming an isotopic ratio of 77 (Wilson \& Rood 1994). A simple LTE analysis of the ${ }^{12} \mathrm{C} /{ }^{13} \mathrm{C}$ line ratio, $R$, indicates that the $\mathrm{HCO}^{+} 1-0$ emission is optically thick at the cloud velocity $(R \sim 11, \tau \sim 7)$ and becomes optically thin in the wings $\left(R \sim 68, \tau \sim 0.3\right.$ at $\left.\sim-2 \mathrm{~km} \mathrm{~s}^{-1}\right)$.

We tentatively fit the profile of the $\mathrm{HCO}^{+} 1-0$ line using the three exponential components as for $\mathrm{CO}$. A good fit is obtained by adopting for the three components the zero velocity intensities summarised in Fig. 3. Similar to what has been found for the CO lines (Lefloch et al. 2012), the bulk of the emission originate in the outflow cavity B1 $\left(g_{2}\right)$, whilst the $g_{1}$ jet component is dominant at high velocities $\left(V<-20 \mathrm{~km} \mathrm{~s}^{-1}\right)$, and the $g_{3}$ component, accounting for the emission from the old cavity B2, contributes only close to systemic velocity. The emission excess detected between $-20 \mathrm{~km} \mathrm{~s}^{-1}$ and $-15 \mathrm{~km} \mathrm{~s}^{-1}$ could be related to the compact clumps along the cavity detected with high-resolution interferometric observations and showing a second emission peak at those velocities (Benedettini et al. 2013). The higher- $J \mathrm{HCO}^{+}$lines are detected with lower signalto-noise, thus the $g_{1}$ component remains undetected and they are fitted by a combination of the $g_{2}$ and $g_{3}$ components. 
L. Podio et al.: Molecular ions in the protostellar shock L1157-B1
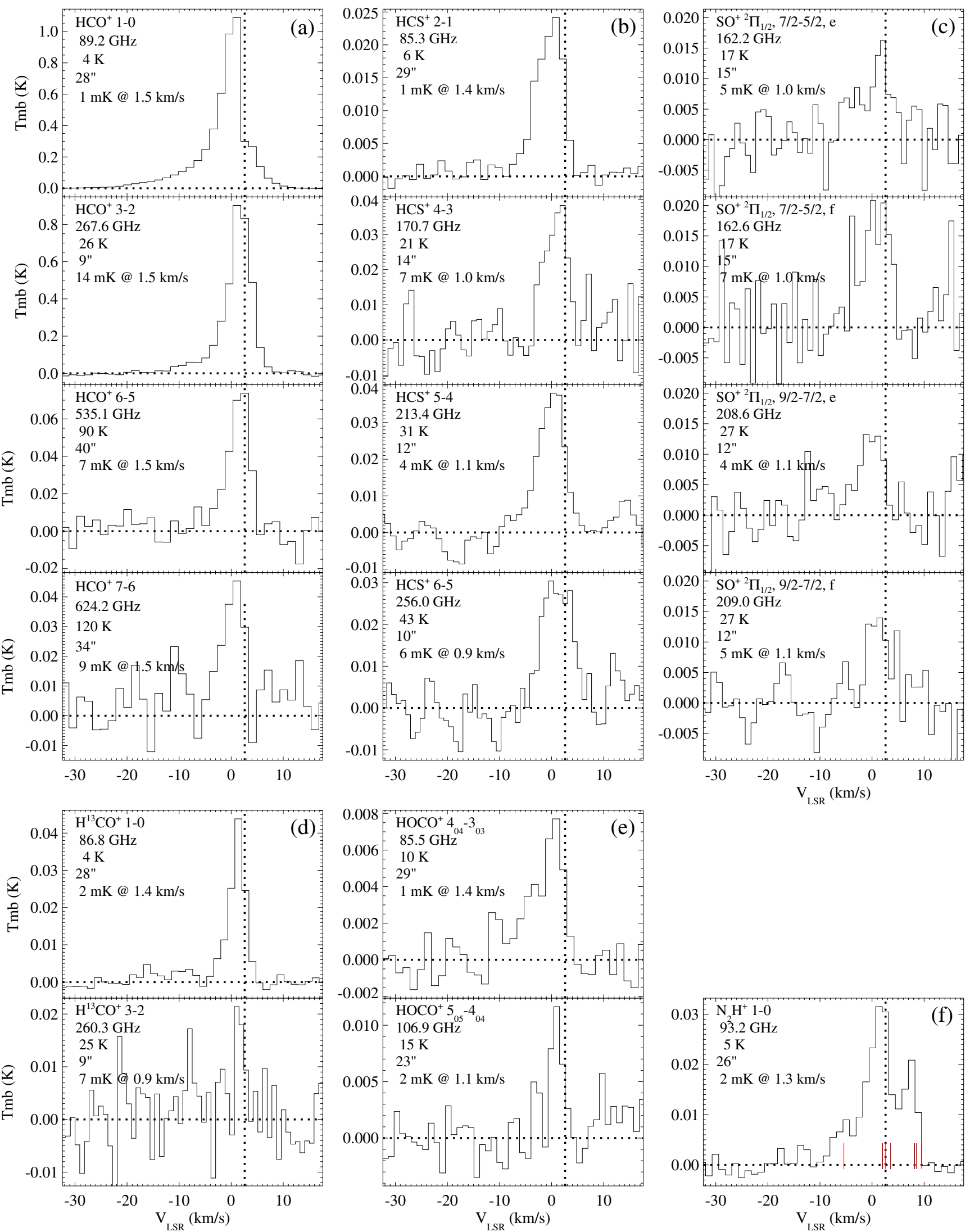

Fig. 2. Line profiles of the molecular ions observed in the $\mathrm{L} 1157-\mathrm{B} 1$ shock: $\mathrm{HCO}^{+}$a), $\left.\left.\mathrm{HCS}^{+} \mathbf{b}\right), \mathrm{SO}^{+} \mathbf{c}\right), \mathrm{H}^{13} \mathrm{CO}^{+}$d), $\left.\mathrm{HOCO}^{+} \mathbf{e}\right)$, and $\left.\mathrm{N}_{2} \mathrm{H}^{+} \mathbf{f}\right)$. The line intensity is in mean beam temperature $\left(T_{\mathrm{mb}}\right)$. The transition, frequency in $\mathrm{GHz}$, upper level energy in $\mathrm{K}$, $\mathrm{HPBW}$ in arcsec, and $1 \sigma \mathrm{rms}$ noise in $\mathrm{mK}$ are indicated. The baseline and the systemic velocity $\left(V_{\mathrm{LSR}}=+2.6 \mathrm{~km} \mathrm{~s}^{-1}\right)$ are indicated by the horizonthal and vertical dotted lines. For $\mathrm{N}_{2} \mathrm{H}^{+} \mathbf{f}$ ), the 15 hyperfine components of the $\mathrm{N}_{2} \mathrm{H}^{+} 1-0$ pattern are indicated by the vertical red lines. (Due to degeneracy only 7 of the 15 hyperfine lines do not overlap in frequency; of these, only the three main groups are resolved because of blending caused by the line broadening.) 
Table 2. Gas physical conditions, column density, and fractional abundances of CO and molecular ions.

\begin{tabular}{lccccc}
\hline \hline Species & $\mathrm{Cmp}$ & $\begin{array}{c}T_{\text {kin }} \\
(\mathrm{K})\end{array}$ & $\begin{array}{c}n_{\mathrm{H}_{2}} \\
\left(\mathrm{~cm}^{-3}\right)\end{array}$ & $\begin{array}{c}N_{\text {species }} \\
\left(\mathrm{cm}^{-2}\right)\end{array}$ & $X=N_{\text {species }} / N_{\mathrm{H}_{2}}$ \\
\hline $\mathrm{CO}^{a}$ & $g_{1}$ & 210 & $\geq 10^{6}$ & $9 \times 10^{15}$ & $10^{-4}$ \\
& $g_{2}$ & $60-80$ & $\geq 10^{5}$ & $9 \times 10^{16}$ & $10^{-4}$ \\
& $g_{3}$ & 20 & $\geq 10^{5}$ & $1 \times 10^{17}$ & $10^{-4}$ \\
$\mathrm{HCO}^{+}$ & $g_{1}$ & $210^{b}$ & $10^{6}-10^{7 b}$ & $1-3 \times 10^{12}$ & $1-3 \times 10^{-8}$ \\
& $g_{2}$ & 60 & $10^{5}$ & $6 \times 10^{12}$ & $7 \times 10^{-9}$ \\
$\mathrm{~N}_{2} \mathrm{H}^{+c}$ & $g_{2} / g_{3}$ & $20-70^{c}$ & - & $0.4-8 \times 10^{12}$ & $0.4-8 \times 10^{-9}$ \\
$\mathrm{HOCO}^{+}$ & $g_{2} / g_{3}$ & 35 & $10^{4}$ & $1 \times 10^{12}$ & $1 \times 10^{-9}$ \\
$\mathrm{SO}^{+d}$ & $g_{2} / g_{3}$ & $25^{d}$ & - & $7 \times 10^{11}$ & $8 \times 10^{-10}$ \\
$\mathrm{HCS}^{+}$ & $g_{2}$ & 80 & $8 \times 10^{5}$ & $6 \times 10^{11}$ & $7 \times 10^{-10}$ \\
& $g_{3}$ & $20^{e}$ & $10^{5 e}$ & $3 \times 10^{11}$ & $3 \times 10^{-10}$ \\
\hline
\end{tabular}

Notes. ${ }^{(a)}$ Lefloch et al. (2012). The fractional abundance of CO is assumed. ${ }^{(b)} T_{\text {kin }}$ and $n_{\mathrm{H}_{2}}$ in $g_{1}$ are assumed to be as derived from CO. ${ }^{(c)} N_{\mathrm{N}_{2} \mathrm{H}^{+}}$, $X_{\mathrm{N}_{2} \mathrm{H}^{+}}$estimated by Codella et al. (2013) assuming LTE at $T_{\text {kin }}=20-70 \mathrm{~K}$ and a source size of $\sim 20-25^{\prime \prime}$ as inferred from CO. (d) Since no collisional coefficients are available, we apply an LTE analysis assuming that the emission originates in the extended outflow cavities $\left(g_{2}, g_{3}\right)$. At difference with the other ions, the estimated temperature is thus an excitation temperature, $T_{\mathrm{ex}}{ }^{(e)} T_{\mathrm{kin}}$ and $n_{\mathrm{H}_{2}}$ in $g_{3}$ are assumed to be as derived from CO. This assumption relies on the similarity of $\mathrm{CO}$ and $\mathrm{HCS}^{+}$profiles at low velocities and on the results of the LVG analysis (see text).

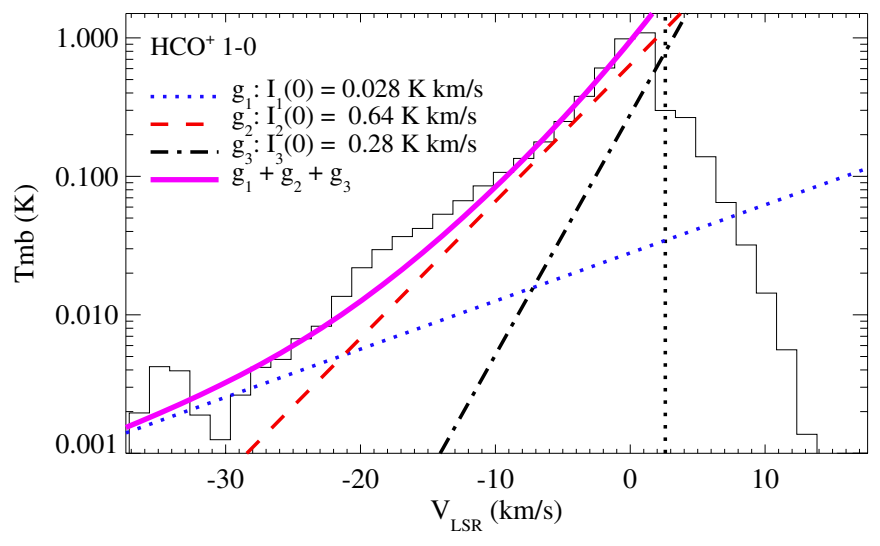

Fig. 3. Profile of the $\mathrm{HCO}^{+} 1-0$ line observed in the L1157-B1 shock. The line intensity is in mean beam temperature $\left(T_{\mathrm{mb}}\right)$ and the systemic velocity $\left(V_{\mathrm{LSR}}=+2.6 \mathrm{~km} \mathrm{~s}^{-1}\right)$ is indicated by the vertical dotted lines. The line profile is fitted by a linear combination of three exponential functions $g_{1}=I_{1}(0) \exp \left(-\left|V / 12.5 \mathrm{~km} \mathrm{~s}^{-1}\right|\right)$ (blue dotted line), $g_{2}=I_{2}(0) \exp \left(-\left|V / 4.4 \mathrm{~km} \mathrm{~s}^{-1}\right|\right)$ (red dashed line), and $g_{3}=I_{3}(0)$ $\exp \left(-\mid V / 2.5 \mathrm{~km} \mathrm{~s}^{-1}\right) \mid$ (dotted-dashed black line). The $\left(g_{1}+g_{2}+g_{3}\right)$ line fit is overplotted (magenta solid line).

We use an LVG model to fit the fluxes of all the $\mathrm{HCO}^{+}$lines, after subtracting the $g_{1}$ component from the $\mathrm{HCO}^{+} 1-0$ line. The flux of the different lines is well accounted for by extended emission from a single gas layer at $T_{\text {kin }} \sim 60 \mathrm{~K}, n_{\mathrm{H}_{2}} \sim 10^{5} \mathrm{~cm}^{-3}$, and a column density $N_{\mathrm{HCO}^{+}} \sim 6 \times 10^{12} \mathrm{~cm}^{-2}$. This solution is in very good agreement with the size and physical conditions found for the outflow cavity B1 ( $g_{2}$ component) for the CO lines (Lefloch et al. 2012, see Table 2). Adopting the CO column density found by these authors, $N_{\mathrm{CO}}=9 \times 10^{16} \mathrm{~cm}^{-2}$, we estimate an $\mathrm{HCO}^{+}$ relative abundance $X_{\mathrm{HCO}^{+}}\left(g_{2}\right)=N_{\mathrm{HCO}^{+}} / N_{\mathrm{H}_{2}} \sim 7 \times 10^{-9}$.

A tentative estimate of the $\mathrm{HCO}^{+}$abundance in the jet component $g_{1}$, can be obtained by assuming the same physical conditions as for $\mathrm{CO}\left(T_{\text {kin }}=210 \mathrm{~K}, n_{\mathrm{H}_{2}} \geq 10^{6} \mathrm{~cm}^{-3}\right)$ and computing the $\mathrm{HCO}^{+}$column density required to account for the $g_{1}$ integrated area. Adopting $\Delta V=10 \mathrm{~km} \mathrm{~s}^{-1}$, we estimate a column density $N_{\mathrm{HCO}^{+}} \sim 1-3 \times 10^{12} \mathrm{~cm}^{-2}$, depending on the assumed density $\left(n_{\mathrm{H}_{2}}=10^{6}-10^{7}\right.$, respectively), implying an
$\mathrm{HCO}^{+}$abundance $X_{\mathrm{HCO}^{+}}\left(g_{1}\right) \sim 1-3 \times 10^{-8}$. The abundance estimated in the $g_{1}$ component agrees within a factor of two with the estimate by Bachiller \& Perez Gutierrez (1997). We recall that the latter is derived using only the $1-0$ line and assuming that the emission is optically thin and thermalized $\left(T_{\text {kin }}=80 \mathrm{~K}\right)$.

Within the uncertainties of the present and previous studies by Bachiller \& Perez Gutierrez (1997), the $\mathrm{HCO}^{+}$abundance estimated both in the jet impact region $\left(g_{1}\right)$ and in the outflow cavities $\left(g_{2}\right)$ can be considered to agree with what estimated at the source position by Bachiller \& Perez Gutierrez (1997) within a factor of a few. This agrees with previous observations of outflows in the $\mathrm{HCO}^{+}$lines showing no evident enhancement of the $\mathrm{HCO}^{+}$abundance both in the outflow cavities and in the high-velocity component with respect to dense core value (Hogerheijde et al. 1998; Tafalla et al. 2010). The chemistry of $\mathrm{HCO}^{+}$and its evolution in the shock is discussed in Sect. 5.

\subsection{2. $\mathrm{N}_{2} \mathrm{H}^{+}$}

A detailed analysis of the detected $\mathrm{N}_{2} \mathrm{H}^{+} 1-0$ line (see Fig. 2f) is presented in Codella et al. (2013). These authors show that the observed line profile is consistent with emission by either the B1 outflow cavity $\left(g_{2}\right)$ and/or with the older and colder B2 cavity $\left(g_{3}\right)$. The peak velocity and line width $\left(V_{\text {peak }} \sim+1.3 \mathrm{~km} \mathrm{~s}^{-1}\right.$, $F W H M \sim 4.3 \mathrm{~km} \mathrm{~s}^{-1}$ ) are in agreement with those found for the other molecular ions. The colum density and fractional abundance $\left(N_{\mathrm{N}_{2} \mathrm{H}^{+}} \sim 0.4-8 \times 10^{12} \mathrm{~cm}^{-2}, X_{\mathrm{N}_{2} \mathrm{H}^{+}} \sim 0.4-8 \times\right.$ $10^{-9}$ ) are derived by assuming LTE conditions at a temperature of $\sim 20-70 \mathrm{~K}$ and an emitting size of $\sim 20-25^{\prime \prime}$ as derived from the analysis of the CO lines by Lefloch et al. (2012). As for $\mathrm{HCO}^{+}, \mathrm{N}_{2} \mathrm{H}^{+}$is also not enhanced in the shock with respect to the on-source value estimated by Bachiller \& Perez Gutierrez (1997). The chemistry of $\mathrm{N}_{2} \mathrm{H}^{+}$is discussed further in Sect. 5.

\subsection{3. $\mathrm{HOCO}^{+}$}

We detect $\mathrm{HOCO}^{+}$emission in the $4_{04}-3_{03}$ and $5_{05}-4_{04}$ lines at 85.5 and $106.9 \mathrm{GHz}$ (see Fig. 2e). So far $\mathrm{HOCO}^{+}$has been detected in different environments, i.e. in the Galactic centre region (Thaddeus et al. 1981; Minh et al. 1988, 1991; Deguchi et al. 2006), in translucent and dark clouds (Turner et al. 1999), 
and around the low-mass Class 0 protostar IRAS $04368+2557$ in L1527 (Sakai et al. 2008). The reported detection, however, is the first one directly associated with a shock spot.

The signal-to-noise of the detected lines is quite low ( 4-6). Similar to the other ions, the profiles favour an origin in the outflow cavities B1 and/or B2 $\left(g_{2}, g_{3}\right)$. However, the lower excitation $\mathrm{HOCO}^{+} 4_{04}-3_{03}$ line at $85.5 \mathrm{GHz}$ also shows a blueshifted wing extending up to $-12 \mathrm{~km} \mathrm{~s}^{-1}$. From an LVG analysis including the two detected lines and the upper limit for the $6_{06}-5_{05}$ line at $128.3 \mathrm{GHz}$, we find that the emission is extended and the best fit is obtained for $n_{\mathrm{H}_{2}} \sim 10^{4} \mathrm{~cm}^{-3}, T_{\text {kin }} \sim 35 \mathrm{~K}$, $N_{\mathrm{HOCO}^{+}} \sim 10^{12} \mathrm{~cm}^{-2}$. Thus, if we consider a CO column density of $\sim 10^{17}\left(g_{2}, g_{3}\right)$, we find $X_{\mathrm{HOCO}^{+}} \sim 10^{-9}$.

The estimated abundance is one to two orders of magnitude more than what is predicted by gas-phase chemistry in cold quiescent clouds (Herbst \& Leung 1986; Turner et al. 1999). Previous studies suggest that $\mathrm{HOCO}^{+}$may be enhanced in shocks and/or UV irradiated regions due to the release of $\mathrm{CO}_{2}$ from dust grain mantles, then reacting with $\mathrm{H}_{3}{ }^{+}$to form $\mathrm{HOCO}^{+}$(Herbst et al. 1977; Minh et al. 1991; Deguchi et al. 2006; Sakai et al. 2008). However, those studies are based on unresolved observations of complex environments and do not allow any conclusion about the origin of $\mathrm{HOCO}^{+}$. Our detection in the L1157-B1 shock, located 69" away from source, is a strong confirmation that $\mathrm{HOCO}^{+}$is an effective shock tracer. The chemistry of $\mathrm{HOCO}^{+}$and the origin of the observed emission are discussed further in Sect. 5.

\subsection{4. $\mathrm{SO}^{+}$}

Figure $2 \mathrm{c}$ shows the $\mathrm{SO}^{+}$transitions detected by our IRAM-30 m observations. To date, $\mathrm{SO}^{+}$lines have been detected in the shocked molecular clump associated with the supernova remnant IC 443G (Turner 1992), in cold clouds and warm active star-forming regions (Turner 1994), and in translucent molecular clouds (Turner 1996). The reported detection is the first detection of $\mathrm{SO}^{+}$emission directly associated with a protostellar shock. We could not detect the $\mathrm{SO}^{+}$doublet at $115.8,116.2 \mathrm{GHz}$ in the $3 \mathrm{~mm}$ band $\left(E_{\mathrm{up}} \sim 9 \mathrm{~K}\right)$ because of the high rms noise (about 13-15 mK). The lines from the higher excitation doublets at $162.2,162.6 \mathrm{GHz}\left(E_{\text {up }} \sim 17 \mathrm{~K}\right)$ and at $208.6,209.0 \mathrm{GHz}$ $\left(E_{\text {up }} \sim 27 \mathrm{~K}\right)$ are detected.

The line profiles suggest that they are dominated by emission from the outflow cavities $\left(g_{2}, g_{3}\right)$ with no evidence of highvelocity emission from the jet impact region $\left(g_{1}\right)$. However, the emission is rather weak and the signal-to-noise ratio is not high enough to firmly conclude anything about the origin of the emission from a simple line profile analysis. Since no collisional coefficients are available, we derive the $\mathrm{SO}^{+}$abundance from the detected line fluxes under the hypothesis of LTE. The $\mathrm{SO}^{+}$abundance estimated under this hypothesis depends on the size of the emitting region, thus we consider two limiting cases.

If we assume that $\mathrm{SO}^{+}$arises from the outflow cavities, either B1 $\left(g_{2}\right)$ or B2 $\left(g_{3}\right)$, the emission is more extended than the beam size, and at first order, the main-beam brightness temperature is a reasonable approximation of the intrinsic source brightness temperature. The relative intensities of the doublets at $162 \mathrm{GHz}$ and $208 \mathrm{GHz}$ imply an excitation temperature of $\sim 25 \mathrm{~K}$. We estimate a column density $N_{\mathrm{SO}^{+}} \sim 7 \times 10^{11} \mathrm{~cm}^{-2}$ and a relative abundance $X_{\mathrm{SO}^{+}} \sim 8 \times 10^{-10}$.

If instead we assume that $\mathrm{SO}^{+}$originates in the compact jet impact region $\left(g_{1} \sim 7-10^{\prime \prime}\right)$, then one has to correct for the source coupling with the telescope beam. We estimate the source coupling factor from an $\mathrm{SiO} J=8-7$ map at $7.4^{\prime \prime}$ resolution obtained at the IRAM-30 m telescope, convolved at the resolution of the different $\mathrm{SO}^{+}$transitions. Our LTE analysis then yields $T_{\mathrm{ex}} \sim 10 \mathrm{~K}$ and a column density $N_{\mathrm{SO}^{+}} \sim 2 \times 10^{12} \mathrm{~cm}^{-2}$, corresponding to a relative abundance $X_{\mathrm{SO}^{+}} \sim 2 \times 10^{-8}$. The derived excitation temperature is much lower than what is estimated from the $\mathrm{CO}$ lines in the $g_{1}$ component by Lefloch et al. (2012) $\left(T_{\mathrm{ex}} \sim 210 \mathrm{~K}\right)$. This suggests that, similar to what is found for the other ions, the bulk of the observed $\mathrm{SO}^{+}$emission originates in the B1, B2 cavities.

To summarise, we estimate an $\mathrm{SO}^{+}$abundance of $\sim 8 \times 10^{-10}$ in the outflow cavities. This value is one to two orders of magnitude higher than what is predicted for cold clouds (Herbst \& Leung 1986), and it favours a shock origin, as suggested by previous detections of large $\mathrm{SO}^{+}$abundances in active star-forming regions (Turner 1994). The chemistry of $\mathrm{SO}^{+}$is discussed in Sect. 5.

\subsection{5. $\mathrm{HCS}^{+}$}

Figure $2 \mathrm{~b}$ shows the $\mathrm{HCS}^{+}$lines detected with the IRAM-30 m telescope. $\mathrm{HCS}^{+}$has been previously detected in molecular clouds (e.g., Ohishi et al. 1992; Sutton et al. 1995), in the lowmass protostar IRAS 16293-2422 (e.g., Schöier et al. 2002), and in association with young sources driving outflows in Cepheus A (Codella et al. 2005). Emission in the bow shock L1157-B1 has already been reported by Bachiller \& Perez Gutierrez (1997) and Yamaguchi et al. (2012). We detected the 2-1, 4-3, 5-4, and 6-5 lines with a signal-to-noise $>4$, whilst only an upper limit is derived for the 7-6 line.

The signal-to-noise ratio is too low to perform a proper line profile analysis. No high-velocity emission associated with the $g_{1}$ component is detected and the lines appear to be dominated by emission from the outflow cavity B1 $\left(g_{2}\right)$. Additional emission by the old B2 cavity $\left(g_{3}\right)$ could contribute close to systemic velocity.

Our LVG calculations confirm that the emission is dominated by the $g_{2}$ component. All the lines but the 2-1 are reproduced well by a single extended component with $T_{\text {kin }} \sim 80 \mathrm{~K}$, $n_{\mathrm{H}_{2}} \sim 8 \times 10^{5} \mathrm{~cm}^{-3}$, and $N_{\mathrm{HCS}^{+}} \sim 6 \times 10^{11} \mathrm{~cm}^{-2}$. The estimated physical conditions are consistent with those found for the B1 outflow cavity (i.e., the $g_{2}$ component), as derived from $\mathrm{CO}$, and indicate an $\mathrm{HCS}^{+}$abundance of $7 \times 10^{-10}$. However, the $\mathrm{HCS}^{+} 2-1$ observed line intensity is about twice as high as the one predicted by the LVG model for the $g_{2}$ component. To account for the intensity of the $J=2-1$, it is necessary to consider an additional component of low excitation. Based on the $\mathrm{HCS}^{+}$2-1 line profile, this component could be associated with the B2 cavity, thus we assume the same physical conditions as derived from $\mathrm{CO}$, i.e. $T_{\mathrm{kin}} \sim 20 \mathrm{~K}, n_{\mathrm{H}_{2}} \sim 10^{5} \mathrm{~cm}^{-3}$. We find that for $N_{\mathrm{HCS}^{+}} \sim 3 \times 10^{11} \mathrm{~cm}^{-2}$ we obtain a good fit of the 2-1 line, whilst the contribution to the higher- $J$ lines is negligible.

To summarise, we find that the $\mathrm{HCS}^{+}$profiles are reproduced well by the emission of the B1 and B2 outflow cavities, with similar abundances $X_{\mathrm{HCS}^{+}}$of 7 and $3 \times 10^{-10}$, respectively. These values are a factor four up to one order of magnitude lower than what estimated by Bachiller \& Perez Gutierrez (1997) based on tentative detections of the 2-1,5-4 lines. As for the other molecular ions, the chemistry of $\mathrm{HCS}^{+}$is discussed in Sect. 5 .

\section{Chemistry of molecular ions}

To understand the origin of the observed molecular ions, we compared the abundances inferred from observations (see 
Table 3. Initial elemental abundances with respect to $\mathrm{H}_{2}$ assumed in our chemical model.

\begin{tabular}{lc}
\hline \hline Species & $X=N_{\text {species }} / N_{\mathrm{H}_{2}}$ \\
\hline $\mathrm{He}$ & 0.14 \\
$\mathrm{~N}$ & $7.40 \times 10^{-5}$ \\
$\mathrm{O}$ & $3.52 \times 10^{-4}$ \\
$\mathrm{C}^{+}$ & $1.46 \times 10^{-4}$ \\
$\mathrm{~S}^{+}$ & $1.60 \times 10^{-7}$ \\
$\mathrm{Si}^{+}$ & $1.60 \times 10^{-8}$ \\
$\mathrm{Fe}^{+}$ & $6.00 \times 10^{-9}$ \\
$\mathrm{Na}^{+}$ & $4.00 \times 10^{-9}$ \\
$\mathrm{Mg}^{+}$ & $1.40 \times 10^{-8}$ \\
\hline
\end{tabular}

Table 2) with predictions obtained via the chemical code Astrochem ${ }^{9}$. Astrochem computes the chemical evolution of a gas at a given temperature, $T_{\text {kin }}$, and hydrogen density, $n_{\mathrm{H}}$. The code reads a network of chemical reactions, builds a system of kinetic rates equations, and solves it using a state-of-the-art, stiff, ordinary differential equation (ODE) solver. It considers gas-phase processes and simple gas-grain interaction, such as freeze-out and desorption via several mechanisms (thermal desorption, cosmic-ray desorption, and photo-desorption). In our calculations we use the OSU ${ }^{10}$ chemical network and assume visual extinction $A_{\mathrm{V}}=10 \mathrm{mag}$ consistent with $\mathrm{C}^{18} \mathrm{O}$ and ${ }^{13} \mathrm{CO}$ column densities, a grain size of $0.1 \mu \mathrm{m}$, and initial elemental abundances as assumed by Wakelam et al. (2006) in their lowmetallicity model with the exception of $\mathrm{He}$ and $\mathrm{N}$, for which we assume cosmic abundances (e.g., Asplund et al. 2005; Tsamis et al. 2011, see Table 3).

As a first step ("STEP 1"), we compute the evolution of the gas in the cloud before the passage of the shock, i.e. for a gas temperature $T_{\text {kin }}=10 \mathrm{~K}$. Figure $4 \mathrm{~A}$ shows the steady-state abundances of the observed molecular ions for gas densities, $n_{\mathrm{H}_{2}}$, between $5 \times 10^{2}$ and $5 \times 10^{5} \mathrm{~cm}^{-3}$, and for three different values of the cosmic rays $(\mathrm{CR})$ ionisation rate $\left(\zeta=3 \times 10^{-17}, 10^{-16}, 3 \times 10^{-16} \mathrm{~s}^{-1}\right)$. The CR ionisation rate in the interstellar medium varies between $10^{-17}$ and a few $10^{-16} \mathrm{~s}^{-1}$ (Padovani et al. 2009). Steady-state abundances are reached on a timescale of $10^{6}-10^{7}$ years, i.e. much longer than the shock kinematical age $\left(t_{\text {shock }} \sim 2000\right.$ years, Gueth et al. 1996). The abundances of $\mathrm{HCO}^{+}, \mathrm{N}_{2} \mathrm{H}^{+}$, and $\mathrm{HOCO}^{+}$are enhanced by a factor of a few up to one order of magnitude for higher CR ionisation rates, whilst they are decreased by 1 to 3 orders of magnitude with increasing density. The dependency of $\mathrm{HCO}^{+}, \mathrm{N}_{2} \mathrm{H}^{+}$, and $\mathrm{HOCO}^{+}$abundance on $\zeta$ and $n_{\mathrm{H}_{2}}$ comes from the main formation process of these ions in the quiescent gas occurring through reactions with $\mathrm{H}_{3}{ }^{+}$:

$$
\begin{aligned}
& \mathrm{H}_{3}{ }^{+}+\mathrm{CO} \rightarrow \mathrm{HCO}^{+}+\mathrm{H}_{2} \\
& \mathrm{H}_{3}{ }^{+}+\mathrm{N}_{2} \rightarrow \mathrm{N}_{2} \mathrm{H}^{+}+\mathrm{H}_{2} \\
& \mathrm{H}_{3}{ }^{+}+\mathrm{CO}_{2} \rightarrow \mathrm{HOCO}^{+}+\mathrm{H}_{2}
\end{aligned}
$$

where $\mathrm{H}_{3}{ }^{+}$is produced by reaction of $\mathrm{H}_{2}$ with $\mathrm{H}_{2}{ }^{+}$, which in turn is produced by cosmic ray ionisation of $\mathrm{H}_{2}$, and destroyed by dissociative recombination with electrons. The abundance of $\mathrm{H}_{3}{ }^{+}$, hence of these molecular ions, therefore increases with $\zeta$ and decreases with density. S-bearing species, $\mathrm{SO}^{+}$and $\mathrm{HCS}^{+}$, are less dependent on the assumed $\zeta$ and stay roughly constant for densities up to $10^{5} \mathrm{~cm}^{-3}$ if $\zeta=1-3 \times 10^{-16} \mathrm{~s}^{-1}$.

\footnotetext{
9 http://smaret.github.io/astrochem/

${ }^{10}$ http://www . physics . ohio-state . edu/ eric/research. html
}

As a second step ("STEP 2"), we compute how the obtained steady-state abundances evolve when the gas is compressed and heated in the shock, i.e. when density and temperature rise from typical dark cloud values $\left(n_{\mathrm{H}_{2}}=10^{4} \mathrm{~cm}^{-3}, T_{\text {kin }}=10 \mathrm{~K}\right)$ to the observed values in the outflow cavity, $g_{2}\left(n_{\mathrm{H}_{2}}=10^{5} \mathrm{~cm}^{-3}\right.$, $\left.T_{\text {kin }}=70 \mathrm{~K}\right)$. Figure $4 \mathrm{~B}$ shows that molecular ion chemistry proceeds fast, so they adjust very rapidly to the change in physical conditions caused by the shock on timescales shorter than or comparable to the shock age ( 2000 years). The abundance of all molecular ions drops owing to the higher density, but this is partially compensated for by the temperature enhancement.

Lastly, the evolution of molecular ions in the shock, i.e. "STEP 2", is recalculated by enhancing (besides the gas temperature and density) the abundance of species that are thought to be sputtered off dust grain mantles, such as $\mathrm{CO}_{2}$ and sulphurbearing species (see Fig. 5). This is done by assuming $\zeta=$ $3 \times 10^{-16} \mathrm{~s}^{-1}$, which is the CR ionisation value that best fits $\mathrm{HCO}^{+}$and $\mathrm{N}_{2} \mathrm{H}^{+}$abundances (see below).

In the following, we discuss how the obtained steady-state abundances in the cloud and their evolution in the shock compare with observations for each of the detected molecular ions. In Figs. 4 and 5, the observed abundances are shown as colour bars covering the range of values inferred for the different components or, when only one abundance value is estimated, the relative uncertainty (see Sect. 4.2). Observations are overplotted at the typical dark cloud density, $n_{\mathrm{H}_{2}}=10^{4} \mathrm{~cm}^{-3}$, in Fig. 4A, and at the shock age, $t_{\text {shock }} \sim 2000$ years, in Figs. 4B and 5 .

\section{$\mathrm{HCO}^{+}$and $\mathrm{N}_{2} \mathrm{H}^{+}$abundances:}

Figure $4 \mathrm{~A}$ shows that the observed $\mathrm{HCO}^{+}$and $\mathrm{N}_{2} \mathrm{H}^{+}$abundances agree with what is predicted in the quiescent gas in the cloud at $n_{\mathrm{H}_{2}}=10^{4} \mathrm{~cm}^{-3}, T_{\text {kin }}=10 \mathrm{~K}$. In the shock, the abundance of $\mathrm{HCO}^{+}$remains roughly constant, as the decrease due to compression is compensated for by the rise in temperature, whilst $\mathrm{N}_{2} \mathrm{H}^{+}$decreases by a factor five up to one order of magnitude for the lowest $\mathrm{CR}$ ionisation rate (Fig. 4B). As a result, $\mathrm{HCO}^{+}$and $\mathrm{N}_{2} \mathrm{H}^{+}$observed abundances $\left(X_{\mathrm{HCO}^{+}} \sim 0.7-3 \times 10^{-8}\right.$, $\left.X_{\mathrm{N}_{2} \mathrm{H}^{+}} \sim 0.4-8 \times 10^{-9}\right)$ are simultaneously reproduced at the shock age only by assuming a high $\mathrm{CR}$ ionisation rate, i.e. $\zeta=1-3 \times 10^{-16} \mathrm{~s}^{-1}$. Owing to recombination, $\mathrm{HCO}^{+}$and $\mathrm{N}_{2} \mathrm{H}^{+}$ abundances are further decreased when neutral molecules are released off the dust grains. Thus, in the following we assume $\zeta=3 \times 10^{-16} \mathrm{~s}^{-1}$ to simultaneously fit the abundances of all the molecular ions (see below). The performed analysis indicates that, in agreement with what is suggested by Codella et al. (2013), $\mathrm{HCO}^{+}$and $\mathrm{N}_{2} \mathrm{H}^{+}$are not enhanced in the shock but instead are a fossil record of the pre-shock gas.

\section{$\mathrm{HOCO}^{+}$abundance:}

In contrast to $\mathrm{HCO}^{+}$and $\mathrm{N}_{2} \mathrm{H}^{+}$, the predicted $\mathrm{HOCO}^{+}$abundance in the cloud is lower than observed by a factor of a few up to two orders of magnitude depending on the assumed $\zeta$ (Fig. 4A). Moreover, the $\mathrm{HOCO}^{+}$abundance is lowered further following the gas compression in the shock and it is one order of magnitude lower than observed, even for the highest $\mathrm{CR}$ ionisation rate $\zeta=3 \times 10^{-16} \mathrm{~s}^{-1}$ (Fig. 4B). As mentioned above, $\mathrm{HOCO}^{+}$is mainly formed via $\mathrm{CO}_{2}$ reacting with $\mathrm{H}_{3}{ }^{+}$ (see Eq. (3)), so it may be a useful probe of $\mathrm{CO}_{2}$, which lacks a permanent electric dipole moment, making radio detection impossible. A low $\mathrm{HOCO}^{+}$abundance is expected in the cloud since most $\mathrm{CO}_{2}$ is trapped on the icy dust mantles. In effect, we find that the steady-state abundance of $\mathrm{CO}_{2}$ in the cloud $\left(n_{\mathrm{H}_{2}}=10^{4} \mathrm{~cm}^{-3}, T_{\text {kin }}=10 \mathrm{~K}, \zeta=3 \times 10^{-16} \mathrm{~s}^{-1}\right)$ is $3 \times 10^{-7}$, 

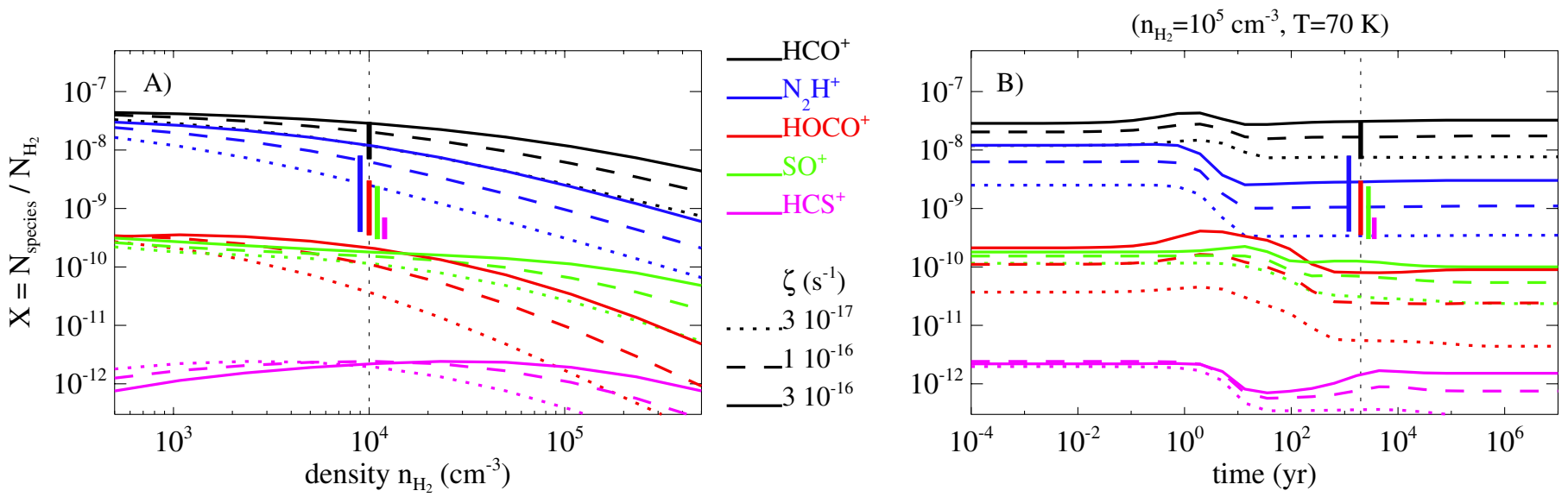

Fig. 4. Observed abundances of molecular ions (colour vertical bars) are compared with theoretical values calculated with Astrochem (colour lines). The colours indicate $\mathrm{HCO}^{+}$(black), $\mathrm{N}_{2} \mathrm{H}^{+}$(blue), $\mathrm{HOCO}^{+}$(red), $\mathrm{SO}^{+}$(green), and $\mathrm{HCS}^{+}$(magenta) abundances. Panel A) "STEP 1": steady-state abundances in the cloud, $X=N_{\text {species }} / N_{\mathrm{H}_{2}}$, as a function of the gas density, $n_{\mathrm{H}_{2}}\left(\mathrm{~cm}^{-3}\right)$, for gas temperature $T_{\mathrm{kin}}=10 \mathrm{~K}$, and cosmic ray ionisation rate $\zeta=3 \times 10^{-17}, 10^{-16}, 3 \times 10^{-16} \mathrm{~s}^{-1}$ (dotted, dashed, and solid lines). Observed abundances are overplotted at the assumed cloud density $\left(n_{\mathrm{H}_{2}}=10^{4} \mathrm{~cm}^{-3}\right.$, vertical dotted line). Panel B) "STEP 2": evolution of steady-state abundances in the shock as a function of time (in years). The gas density and temperature are raised from cloud values $\left(n_{\mathrm{H}_{2}}=10^{4} \mathrm{~cm}^{-3}, T_{\text {kin }}=10 \mathrm{~K}\right)$ to the observed values in the outflow cavity $\left(n_{\mathrm{H}_{2}}=10^{5} \mathrm{~cm}^{-3}, T_{\text {kin }}=70 \mathrm{~K}\right)$. Observed abundances are overplotted at the shock age ( $t_{\text {shock }} \sim 2000$ years, vertical dotted line).

in agreement with what is predicted by other chemical models (e.g., Lee et al. 1996) and what has been measured towards various star-forming regions through observations of the $15 \mu \mathrm{m}$ band (van Dishoeck et al. 1996; Dartois et al. 1998; Boonman et al. 2003). Based on $\mathrm{HOCO}^{+}$observations in the Galactic centre, Minh et al. (1991) first suggested that a high $\mathrm{HOCO}^{+}$abundance may be caused by shocks, which release $\mathrm{CO}_{2}$ molecules frozen on the mantles of dust grains into gas-phase via graingrain (shattering) or gas-grain (sputtering) collisions and/or via evaporation by UV-photons in dissociative shocks.

We investigate this scenario with Astrochem by computing the evolution of $\mathrm{HOCO}^{+}$in the shock when enhancing $\mathrm{CO}_{2}$ with respect to its steady-state abundance. We have direct evidence of dust grains sputtering in the $\mathrm{B} 1$ shock from $\mathrm{SiO}$ observations (Gueth et al. 1998; Nisini et al. 2007). Figure 5C shows that $\mathrm{HOCO}^{+}$is rapidly enhanced by more than one order of magnitude for increasing $X_{\mathrm{CO}_{2}}$, and it stays high for $\sim 10^{4}$ years. The observed value, $X_{\mathrm{HOCO}^{+}} \sim 10^{-9}$, is matched at the shock age ( $t_{\text {shock }} \sim 2000$ years) for $X_{\mathrm{CO}_{2}} \geq 2 \times 10^{-5}$, i.e. around two orders of magnitude higher than in the cloud. This value is consistent with previous estimates of the amount of solid $\mathrm{CO}_{2}$ trapped in the icy mantle of dust grains (e.g., D'Hendecourt \& Jourdain de Muizon 1989; Tielens et al. 1991; de Graauw et al. 1996). Thus, this result may indicate total evaporation of the ice mantles, in agreement with the water abundance estimated by Busquet et al. (2014) in the hot shock component.

The abundance of the other molecular ions is not (for $\mathrm{HCO}^{+}$) or only slightly (by a factor lower than two for the other molecular ions) affected by the $\mathrm{CO}_{2}$ enhancement in the shock.

\section{$\mathrm{SO}^{+}$abundance:}

In agreement with what has been estimated by previous gasphase chemistry models (e.g., Herbst \& Leung 1986), our chemical model predicts an $\mathrm{SO}^{+}$steady-state abundance in the cloud of $1.8 \times 10^{-10}$, which is lowered down to $1 \times 10^{-10}$ in the shock. This is lower than observed by around one order of magnitude. On the other hand, Neufeld \& Dalgarno (1989) have shown that the abundance of $\mathrm{SO}^{+}$is strongly enhanced in shocks due to the release of sulphur from dust mantles and its subsequent ionisation, as $\mathrm{SO}^{+}$is primarly formed via $\mathrm{S}^{+}+\mathrm{OH} \rightarrow \mathrm{SO}^{+}+\mathrm{H}$.

We explore this scenario by computing the evolution of molecular ions in the shock when enhancing the abundance of atomic $\mathrm{S}$ or $\mathrm{H}_{2} \mathrm{~S}$ (Figs. 5D and E, respectively). $\mathrm{S}$ and $\mathrm{H}_{2} \mathrm{~S}$ are enhanced up to values that are larger than the initial abundance of $\mathrm{S}^{+}$in the model, since most of $\mathrm{S}$ is assumed to be initially trapped on dust grains. However, they are still consistent with the solar abundance of S (e.g., Asplund et al. 2005). Following our analysis of $\mathrm{HOCO}^{+}$we also set $X_{\mathrm{CO}_{2}}=6 \times 10^{-5}$. Figure 5 shows that, if the released $\mathrm{S}$ or $\mathrm{H}_{2} \mathrm{~S}$ are $\sim 2 \times 10^{-6}$ and $\geq 2 \times 10^{-7}$, respectively, $\mathrm{SO}^{+}$is rapidly (less than 10 years) enhanced behind the shock and its abundance remains high on large timescales, matching the observed value $\left(X_{\mathrm{SO}^{+}} \sim 8 \times 10^{-10}\right)$ at the shock age. This confirms a shock origin for $\mathrm{SO}^{+}$.

The amount of $\mathrm{H}_{2} \mathrm{~S}$ that needs to be released in gas phase to reproduce the observed $\mathrm{SO}^{+}$abundance is in agreement with the $\mathrm{H}_{2} \mathrm{~S}$ abundance in the $\mathrm{B} 1$ shock estimated by Bachiller \& Perez Gutierrez (1997) $\left(X_{\mathrm{H}_{2} \mathrm{~S}} \sim 3 \times 10^{-7}\right)$. Concerning the other molecular ions we note that the abundances of $\mathrm{N}_{2} \mathrm{H}^{+}$and $\mathrm{HOCO}^{+}$are not very affected by $\mathrm{S}$ or $\mathrm{H}_{2} \mathrm{~S}$ enhancement, whilst the abundance of $\mathrm{HCO}^{+}$is lowered by a factor lower than two, still matching the observed value. $\mathrm{HCS}^{+}$is also enhanced but not enough to match the observed abundance (see below).

\section{$\mathrm{HCS}^{+}$abundance:}

Figure 4 shows that the $\mathrm{HCS}^{+}$abundance in the cloud is very low $\sim 10^{-12}$, even for high CR ionisation rates, and it remains roughly unaltered after the passage of the shock. The abundance of $\mathrm{HCS}^{+}$is strongly related to that of $\mathrm{CS}$, since this ion is formed when $\mathrm{CS}$ reacts with $\mathrm{HCO}^{+}, \mathrm{H}_{3}{ }^{+}$, and $\mathrm{H}_{3} \mathrm{O}^{+}$, whilst the main destructive mechanism is dissociative recombination into $\mathrm{CS}$. Therefore, the low values of $X_{\mathrm{HCS}^{+}}$in the cloud are likely due to a lack of CS. Indeed, the steady-state abundance of CS in the cloud $\left(n_{\mathrm{H}_{2}}=10^{4} \mathrm{~cm}^{-3}, T_{\text {kin }}=10 \mathrm{~K}, \zeta=3 \times 10^{-16} \mathrm{~s}^{-1}\right)$ is of $\sim 10^{-10}$, which is three orders of magnitude lower than what was estimated in the B1 shock by Bachiller \& Perez Gutierrez (1997) $\left(X_{\mathrm{CS}} \sim 2 \times 10^{-7}\right)$. 
STEP 2: Evolution at SHOCK conditions $\left(\mathrm{n}_{\mathrm{H}_{2}}=10^{5} \mathrm{~cm}^{-3}, \mathrm{~T}=70 \mathrm{~K}\right)$

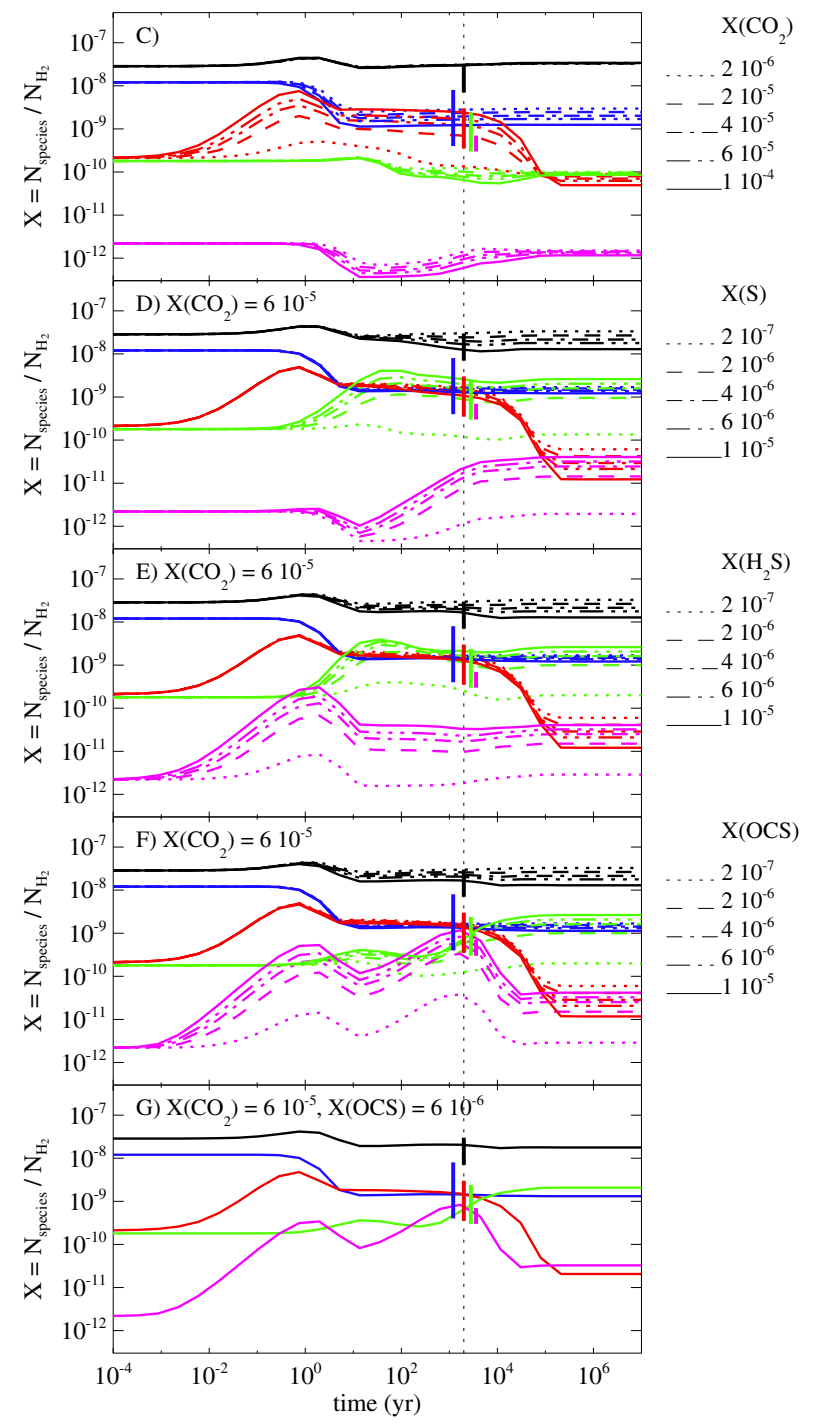

Fig. 5. Evolution of molecular ions abundances in the shock as a function of time ("STEP 2"). The colours indicate $\mathrm{HCO}^{+}$(black), $\mathrm{N}_{2} \mathrm{H}^{+}$(blue), $\mathrm{HOCO}^{+}$(red), $\mathrm{SO}^{+}$(green), and $\mathrm{HCS}^{+}$(magenta) abundances. Observed abundances (colour vertical bars) are overplotted at the shock age $\left(t_{\text {shock }} \sim 2000\right.$ years, vertical dotted line $)$. The evolution is computed from steady-state values in the cloud $\left(n_{\mathrm{H}_{2}}=10^{4}\right.$ $\mathrm{cm}^{-3}, T_{\text {kin }}=10 \mathrm{~K}, \zeta=3 \times 10^{-16} \mathrm{~s}^{-1}$ ) by enhancing the gas temperature and density $\left(n_{\mathrm{H}_{2}}=10^{5} \mathrm{~cm}^{-3}, T_{\text {kin }}=70 \mathrm{~K}\right)$, and the abundance of molecules which are thought to be sputtered off dust grain mantles: $\mathrm{CO}_{2}, \mathrm{~S}, \mathrm{H}_{2} \mathrm{~S}$, and OCS (panels $\left.\left.\mathbf{C}\right), \mathbf{D}\right), \mathbf{E}$ ), and $\mathbf{F}$ ), respectively). When varying $\mathrm{S}, \mathrm{H}_{2} \mathrm{~S}$, and OCS abundances, $X_{\mathrm{CO}_{2}}$ is set to $=6 \times 10^{-5}$. Finally, panel $\mathbf{G}$ ) shows the evolution of molecular ions abundances in our "best-fit model", i.e. for $X_{\mathrm{CO}_{2}}=6 \times 10^{-5}$ and $X_{\mathrm{OCS}}=6 \times 10^{-6}$.

Figures $5 \mathrm{D}$ and $\mathrm{E}$ show that $\mathrm{HCS}^{+}$is enhanced by almost two orders of magnitude when $\mathrm{S}$ or $\mathrm{H}_{2} \mathrm{~S}$ are released off the dust grains but is still lower than observed by one order of magnitude. However, larger CS and $\mathrm{HCS}^{+}$abundances can be obtained if we assume that OCS is one of the main sulphur carriers on dust grains, as suggested by Wakelam et al. $(2004,2005)$ and Codella et al. (2005). In particular, Fig. 5F shows that the observed $\mathrm{HCS}^{+}$ abundance $\left(X_{\mathrm{HCS}^{+}} \sim 3-7 \times 10^{-10}\right)$ is matched at the shock age when the abundance of OCS in the shock is enhanced up to values $\geq 2 \times 10^{-6}$. Interestingly, $X_{\mathrm{CS}}$ also turns out to agree with the
Table 4. Abundances of non-detected molecular ions with respect to $\mathrm{H}_{2}$, as obtained from our "best-fit model" (see Fig. 5G).

\begin{tabular}{lc}
\hline \hline Species & $X=N_{\text {species }} / N_{\mathrm{H}_{2}}$ \\
\hline $\mathrm{CO}^{+}$ & $6 \times 10^{-14}$ \\
$\mathrm{H}_{2} \mathrm{O}^{+}$ & $7 \times 10^{-13}$ \\
$\mathrm{H}_{3} \mathrm{O}^{+}$ & $8 \times 10^{-9}$ \\
$\mathrm{CH}^{+}$ & $6 \times 10^{-16}$ \\
$\mathrm{HOC}^{+}$ & $1 \times 10^{-11}$ \\
$\mathrm{HCNH}^{+}$ & $4 \times 10^{-11}$ \\
$\mathrm{NH}_{4}^{+}$ & $9 \times 10^{-11}$ \\
$\mathrm{CN}^{-}$ & $2 \times 10^{-18}$ \\
$\mathrm{C}_{3} \mathrm{~N}^{-}$ & $3 \times 10^{-18}$ \\
$\mathrm{C}_{4} \mathrm{H}^{-}$ & $1 \times 10^{-16}$ \\
\hline
\end{tabular}

observed value within a factor two $\left(X_{\mathrm{CS}} \sim 2 \times 10^{-7}\right.$, Bachiller $\&$ Perez Gutierrez 1997).

On the other hand, we note that if the amount of OCS released off the dust grains is $\geq 2 \times 10^{-6}$, the OCS abundance at the shock age is $\sim 10^{-6}$, and it decreases down to a value of $\sim 10^{-7}$ only on timescales $\geq 10^{4}$ years. Thus, $X_{\mathrm{OCS}}$ at the shock age is around one to two orders of magnitude larger than what estimated in the B1-B2 cavities by Bachiller \& Perez Gutierrez (1997) $\left(X_{\mathrm{OCS}} \sim 3 \times 10^{-8}-2 \times 10^{-7}\right)$. In this regard, several authors point out that at present chemical models are unable to explain $\mathrm{CS}, \mathrm{HCS}^{+}$, and OCS abundances simultaneously and that the chemistry of sulphur-bearing species is not fully understood (Doty et al. 2004; Wakelam et al. 2004, 2005). Doty et al. (2004) point out that the rates of ion-molecule reactions producing $\mathrm{HCS}^{+}$, i.e. $\left(\mathrm{HCO}^{+}, \mathrm{H}_{3}{ }^{+}, \mathrm{H}_{3} \mathrm{O}^{+}\right)+\mathrm{CS}$, have only been estimated but would need to be confirmed by laboratory experiments.

"Best-fit model":

Figure 5 shows that the abundances of the detected molecular ions (see Table 2) are reproduced well by the gas evolution in the shock if $\mathrm{CO}_{2}, \mathrm{~S}$, and OCS are substantially enhanced by dust grain sputtering and/or evaporation. In particular, we are able to simultaneously match all the estimated abundances at the shock age $(\sim 2000$ years $)$ if we assume $X_{\mathrm{CO}_{2}}=6 \times 10^{-5}$ and $X_{\mathrm{OCS}}=6 \times 10^{-6}$ (see Fig. $5 \mathrm{G}$ ). To verify the robustness of our "best-fit model", we verify that the abundances of other species, both ionic and neutral, are consistent with present and past observations. The abundances obtained at the shock age of molecular ions and anions that are not detected in our survey are summarised in Table 4 . They are all $<10^{-10}$, hence consistent with non-detection of the transitions falling in the spectral range covered by our survey, with the exception of $\mathrm{H}_{3} \mathrm{O}^{+}$, whose predicted abundance is $8 \times 10^{-9}$. The only $\mathrm{H}_{3} \mathrm{O}^{+}$transition covered by our survey is the transition at $307.2 \mathrm{GHz}$, observed with WILMA with a resolution of $2 \mathrm{MHz}$, for which we derive an upper limit of $0.03 \mathrm{~K} \mathrm{~km} \mathrm{~s}^{-1}$. This is consistent with non-detection when assuming the gas temperature and density inferred for the $g_{2}$ component.

\section{Conclusions}

In this paper we presented a comprehensive study of the chemistry of molecular ions in protostellar shocks. As part of the ASAI and CHESS projects, we performed a census of molecular ions in the chemically rich protostellar shock L1157-B1 by means of an unbiased and high-sensitivity $(\sim 1.5 \mathrm{mK}$ in the $3 \mathrm{~mm}$ 
band) spectral survey executed with the IRAM-30 m telescope and Herschel/HIFI. From our analysis we obtained the following results:

- We report emission from $\mathrm{HCO}^{+}, \mathrm{N}_{2} \mathrm{H}^{+}, \mathrm{HCS}^{+}$, and for the first time in a protostellar shock, from $\mathrm{HOCO}^{+}$and $\mathrm{SO}^{+}$.

- All the detected lines peak at blue-shifted velocity, $\sim 0.5-3 \mathrm{~km} \mathrm{~s}^{-1}$ with respect to systemic $\left(V_{\mathrm{LSR}}=+2.6 \mathrm{~km} \mathrm{~s}^{-1}\right)$ and have a line width of $\sim 3-7 \mathrm{~km} \mathrm{~s}^{-1}$. A higher velocity component ( $V$ up to $-40 \mathrm{~km} \mathrm{~s}^{-1}$ ) associated with the primary jet is detected in the $\mathrm{HCO}^{+} 1-0$ line.

- The lines profiles can be decomposed into different velocity components following an exponential intensity-velocity relation as found for the CO lines (Lefloch et al. 2012). The bulk of the emission is associated with the extended outflow cavities and originates in a gas with temperature $T_{\text {kin }} \sim 20-70 \mathrm{~K}$ and density $n_{\mathrm{H}_{2}} \sim 10^{5} \mathrm{~cm}^{-3}$, in agreement with what is found for the $\mathrm{CO}$ lines.

- Inferred $\mathrm{HCO}^{+}$and $\mathrm{N}_{2} \mathrm{H}^{+}$abundances $\left(X_{\mathrm{HCO}^{+}} \sim 0.7-3 \times\right.$ $\left.10^{-8}, X_{\mathrm{N}_{2} \mathrm{H}^{+}} \sim 0.4-8 \times 10^{-9}\right)$ are in agreement with onsource values (Bachiller \& Perez Gutierrez 1997), suggesting that these species are not enhanced in the shock. This is further confirmed by the fact that the observed abundances agree with steady-state abundances in the cloud (i.e., at $T_{\text {kin }}=10 \mathrm{~K}, n_{\mathrm{H}_{2}}=10^{4} \mathrm{~cm}^{-3}$ ). The decrease in their abundance due to gas compression in the shock is compensated for by the rise in temperature, and predicted values match the observations for the high cosmic ray ionisation rate $\zeta=3 \times 10^{-16} \mathrm{~s}^{-1}$. Therefore, $\mathrm{HCO}^{+}$and $\mathrm{N}_{2} \mathrm{H}^{+}$are a fossil record of the gas chemistry before the arrival of the shock.

- $\mathrm{HOCO}^{+}, \mathrm{SO}^{+}$, and $\mathrm{HCS}^{+}$abundances $\left(X_{\mathrm{HOCO}^{+}} \sim 10^{-9}\right.$, $\left.X_{\mathrm{SO}^{+}} \sim 8 \times 10^{-10}, X_{\mathrm{HCS}^{+}} \sim 3-7 \times 10^{-10}\right)$, instead, are one to two orders of magnitude larger than predicted steady-state abundances in the cloud. On the other hand, they are strongly enhanced in the shock if $\mathrm{CO}_{2}, \mathrm{~S}, \mathrm{H}_{2} \mathrm{~S}$, and OCS abundances are increased with respect to steady-state due to release off the dust grains in the shock. As suggested by previous studies (e.g., Minh et al. 1991; Deguchi et al. 2006; Turner 1992, 1994), these species are effective shock tracers.

- The observed abundances allow constraining the amount of $\mathrm{CO}_{2}$ and sulphur-bearing species released in the shock. To reproduce $\mathrm{HOCO}^{+}$observations, $\mathrm{CO}_{2}$ should be enhanced up to abundances $\geq 2 \times 10^{-5}$, i.e. two orders of magnitude greater than its gas-phase abundance in the quiescent gas. This agrees with previous estimate of the $\mathrm{CO}_{2}$ content on dust mantles. To reproduce $\mathrm{SO}^{+}$, instead, the sulphur released in the form of $\mathrm{H}_{2} \mathrm{~S}$ molecules should be $\geq 2 \times 10^{-7}$. This value agrees with the $\mathrm{H}_{2} \mathrm{~S}$ abundance in $\mathrm{B} 1$ estimated by Bachiller \& Perez Gutierrez (1997).

- As noted in previous studies, CS and $\mathrm{HCS}^{+}$abundances in the quiescent gas in dark clouds are very low. However, the observed $\mathrm{HCS}^{+}$and $\mathrm{CS}$ abundances $\left(X_{\mathrm{HCS}^{+}} \sim 3-7 \times 10^{-10}\right.$, $X_{\mathrm{CS}} \sim 2 \times 10^{-7}$ ) are matched if OCS is assumed to be one of the main sulphur carriers on dust grains, as suggested by Wakelam et al. $(2004,2005)$ and Codella et al. (2005), and its abundance is enhanced to values $\geq 2 \times 10^{-6}$ in the shock. Such high $X_{\mathrm{OCS}}$ values, however, are at least one order of magnitude higher than estimated by Bachiller \& Perez Gutierrez (1997) and are lowered to the observed values only on timescales $\geq 10^{4}$ years, i.e. much greater than the shock age $\left(t_{\text {shock }} \sim 2000\right.$ years $)$. This difficulty in simultaneously reproducing $\mathrm{CS}, \mathrm{HCS}^{+}$, and OCS abundances has already been reported by other authors, and it indicates that the chemistry of sulphur-bearing species has not yet been fully understood (Doty et al. 2004; Wakelam et al. 2004, 2005). In this regard, laboratory experiments are required to verify the rates of ion-molecule reactions producing $\mathrm{HCS}^{+}$, i.e. $\left(\mathrm{HCO}^{+}, \mathrm{H}_{3}{ }^{+}, \mathrm{H}_{3} \mathrm{O}^{+}\right)+\mathrm{CS}$.

Acknowledgements. L. Podio acknowledges the funding from the FP7 IntraEuropean Marie Curie Fellowship (PIEF-GA-2009-253896). B. Lefloch and C. Ceccarelli acknowledge financial support by the French space agency CNES. R. Bachiller acknowledges partial support from the Spanish MINECO under grant FIS2012-32096.

\section{References}

Asplund, M., Grevesse, N., \& Sauval, A. J. 2005, in Cosmic Abundances as Records of Stellar Evolution and Nucleosynthesis, eds. T. G. Barnes, III, \& F. N. Bash, ASP Conf. Ser., 336, 25

Bachiller, R., \& Perez Gutierrez, M. 1997, ApJ, 487, L93

Bachiller, R., Pérez Gutiérrez, M., Kumar, M. S. N., \& Tafalla, M. 2001, A\&A 372, 899

Benedettini, M., Viti, S., Codella, C., et al. 2007, MNRAS, 381, 1127

Benedettini, M., Busquet, G., Lefloch, B., et al. 2012, A\&A, 539, L3

Benedettini, M., Viti, S., Codella, C., et al. 2013, MNRAS, 436, 179

Boonman, A. M. S., van Dishoeck, E. F., Lahuis, F., \& Doty, S. D. 2003, A\&A, 399,1063

Busquet, G., Lefloch, B., Benedettini, M., et al. 2014, A\&A, 561, A120

Caselli, P., Walmsley, C. M., Terzieva, R., \& Herbst, E. 1998, ApJ, 499, 234

Caselli, P., Benson, P. J., Myers, P. C., \& Tafalla, M. 2002, ApJ, 572, 238

Ceccarelli, C., \& CHESS Consortium. 2010, in 38th COSPAR Scientific Assembly, COSPAR Meeting, 38, 2476

Codella, C., Bachiller, R., Benedettini, M., et al. 2005, MNRAS, 361, 244

Codella, C., Benedettini, M., Beltrán, M. T., et al. 2009, A\&A, 507, L25

Codella, C., Lefloch, B., Ceccarelli, C., et al. 2010, A\&A, 518, L112

Codella, C., Ceccarelli, C., Bottinelli, S., et al. 2012a, ApJ, 744, 164

Codella, C., Ceccarelli, C., Lefloch, B., et al. 2012b, ApJ, 757, L9

Codella, C., Viti, S., Ceccarelli, C., et al. 2013, ApJ, 776, 52

Dartois, E., D'Hendecourt, L., Boulanger, F., et al. 1998, A\&A, 331, 651

Davis, C. J., \& Eisloeffel, J. 1995, A\&A, 300, 851

de Graauw, T., Helmich, F. P., Phillips, T. G., et al. 2010, A\&A, 518, L6

de Graauw, T., Whittet, D. C. B., Gerakines, P. A., et al. 1996, A\&A, 315, L345

Deguchi, S., Miyazaki, A., \& Minh, Y. C. 2006, PASJ, 58, 979

D'Hendecourt, L. B., \& Jourdain de Muizon, M. 1989, A\&A, 223, L5

Doty, S. D., Schöier, F. L., \& van Dishoeck, E. F. 2004, A\&A, 418, 1021

Dubernet, M.-L., Alexander, M. H., Ba, Y. A., et al. 2013, A\&A, 553, A50

Flower, D. R. 1999, MNRAS, 305, 651

Girart, J. M., Ho, P. T. P., Rudolph, A. L., et al. 1999, ApJ, 522, 921

Girart, J. M., Viti, S., Williams, D. A., Estalella, R., \& Ho, P. T. P. 2002, A\&A, 388,1004

Gueth, F. Guilloteau, S. \& Bachiller, R. 1996, A\&A, 307, 891

Gueth, F., Guilloteau, S., \& Bachiller, R. 1998, A\&A, 333, 287

Hammami, K., Lique, F., Jaïdane, N., et al. 2007, A\&A, 462, 789

Herbst, E., \& Leung, C. M. 1986, MNRAS, 222, 689

Herbst, E., Green, S., Thaddeus, P., \& Klemperer, W. 1977, ApJ, 215, 503

Hogerheijde, M. R., van Dishoeck, E. F., Blake, G. A., \& van Langevelde, H. J. 1998, ApJ, 502, 315

Lee, H.-H., Bettens, R. P. A., \& Herbst, E. 1996, A\&AS, 119, 111

Lee, C.-F., Ho, P. T. P., Hirano, N., et al. 2007, ApJ, 659, 499

Lefloch, B., Cabrit, S., Codella, C., et al. 2010, A\&A, 518, L113

Lefloch, B., Cabrit, S., Busquet, G., et al. 2012, ApJ, 757, L25

Looney, L. W., Tobin, J. J., \& Kwon, W. 2007, ApJ, 670, L131

Minh, Y. C., Irvine, W. M., \& Ziurys, L. M. 1988, ApJ, 334, 175

Minh, Y. C., Brewer, M. K., Irvine, W. M., Friberg, P., \& Johansson, L. E. B. 1991, A\&A, 244, 470

Monteiro, T. 1984, MNRAS, 210, 1

Müller, H. S. P., Thorwirth, S., Roth, D. A., \& Winnewisser, G. 2001, A\&A, 370, L49

Neufeld, D. A., \& Dalgarno, A. 1989, ApJ, 340, 869

Nisini, B., Codella, C., Giannini, T., et al. 2007, A\&A, 462, 163

Nisini, B., Benedettini, M., Codella, C., et al. 2010, A\&A, 518, L120

Ohishi, M., Irvine, W. M., \& Kaifu, N. 1992, in Astrochemistry of Cosmic Phenomena, ed. P. D. Singh, IAU Symp., 150, 171

Padovani, M., Walmsley, C. M., Tafalla, M., Galli, D., \& Müller, H. S. P. 2009, A\&A, 505, 1199

Pickett, H. M., Poynter, R. L., Cohen, E. A., et al. 1998, J. Quant. Spectrosc. Rad. Transf., 60, 883 
Pilbratt, G. L., Riedinger, J. R., Passvogel, T., et al 2010, A\&A, 518, L1 Rawlings, J. M. C., Redman, M. P., Keto, E., \& Williams, D. A. 2004, MNRAS, 351,1054

Roelfsema, P. R., Helmich, F. P., Teyssier, D., et al. 2012, A\&A, 537, A17

Sakai, N., Sakai, T., Aikawa, Y., \& Yamamoto, S. 2008, ApJ, 675, L89

Schöier, F. L., Jørgensen, J. K., van Dishoeck, E. F., \& Blake, G. A. 2002, A\&A, 390, 1001

Sutton, E. C., Peng, R., Danchi, W. C., et al. 1995, ApJS, 97, 455

Tafalla, M., \& Bachiller, R. 1995, ApJ, 443, L37

Tafalla, M., Santiago-García, J., Hacar, A., \& Bachiller, R. 2010, A\&A, 522, A91

Tappe, A., Forbrich, J., Martín, S., Yuan, Y., \& Lada, C. J. 2012, ApJ, 751, 9

Thaddeus, P., Guelin, M. \& Linke, R. A. 1981, ApJ, 246, L41

Tielens, A. G. G. M., Tokunaga, A. T., Geballe, T. R., \& Baas, F. 1991, ApJ, 381 , 181
Tsamis, Y. G., Walsh, J. R., Vílchez, J. M., \& Péquignot, D. 2011, MNRAS, 412, 1367

Turner, B. E. 1992, ApJ, 396, L107

Turner, B. E. 1994, ApJ, 430, 727

Turner, B. E. 1996, ApJ, 468, 694

Turner, B. E., Terzieva, R., \& Herbst, E. 1999, ApJ, 518, 699

van Dishoeck, E. F., Helmich, F. P., de Graauw, T., et al. 1996, A\&A, 315, L349

Vasta, M. Codella, C., Lorenzani, A., et al. 2012, A\&A, 537, A98

Viti, S., Natarajan, S., \& Williams, D. A. 2002, MNRAS, 336, 797

Wakelam, V., Caselli, P., Ceccarelli, C., Herbst, E., \& Castets, A. 2004, A\&A, 422,159

Wakelam, V., Ceccarelli, C., Castets, A., et al. 2005, A\&A, 437, 149

Wakelam, V., Herbst, E., Selsis, F., \& Massacrier, G. 2006, A\&A, 459, 813

Wilson, T. L., \& Rood, R. 1994, ARA\&A, 32, 191

Yamaguchi, T., Takano, S., Watanabe, Y., et al. 2012, PASJ, 64, 105 